ARTICLE

https://doi.org/10.1038/s41467-020-19786-7

\title{
TDP-43 interacts with amyloid- $\beta$, inhibits fibrillization, and worsens pathology in a model of Alzheimer's disease
}

\author{
Yao-Hsiang Shih (10) 1,2,3,8, Ling-Hsien Tu (1) 1,4,8, Ting-Yu Chang ${ }^{1,5}$, Kiruthika Ganesan¹, Wei-Wei Chang ${ }^{1}$, \\ Pao-Sheng Chang (1) 1, Yu-Sheng Fang ${ }^{1,6}$, Yeh-Tung Lin ${ }^{1,5}$, Lee-Way $\operatorname{Jin}^{7}$ \& Yun-Ru Chen (1) 1,5,6凶
}

TDP-43 inclusions are found in many Alzheimer's disease (AD) patients presenting faster disease progression and greater brain atrophy. Previously, we showed full-length TDP-43 forms spherical oligomers and perturbs amyloid- $\beta(A \beta)$ fibrillization. To elucidate the role of TDP-43 in AD, here, we examined the effect of TDP-43 in A $\beta$ aggregation and the attributed toxicity in mouse models. We found TDP-43 inhibited $A \beta$ fibrillization at initial and oligomeric stages. A $\beta$ fibrillization was delayed specifically in the presence of $\mathrm{N}$-terminal domain containing TDP-43 variants, while C-terminal TDP-43 was not essential for A $\beta$ interaction. TDP43 significantly enhanced $A \beta^{\prime} s$ ability to impair long-term potentiation and, upon intrahippocampal injection, caused spatial memory deficit. Following injection to AD transgenic mice, TDP-43 induced inflammation, interacted with $A \beta$, and exacerbated AD-like pathology. TDP-43 oligomers mostly colocalized with intracellular $A \beta$ in the brain of AD patients. We conclude that TDP-43 inhibits $A \beta$ fibrillization through its interaction with $A \beta$ and exacerbates $A D$ pathology.

\footnotetext{
${ }^{1}$ Genomics Research Center, Academia Sinica, 128, Academia Road, Section 2, Nankang District, Taipei 115, Taiwan. ${ }^{2}$ Department of Anatomy, School of Medicine, Kaohsiung Medical University, 100, Shih-Chuan 1st Road, Sanmin District, Kaohsiung 80708, Taiwan. ${ }^{3}$ Department of Medical Research, Kaohsiung Medical University Hospital, 100, Tzyou 1st Road, Sanmin District, Kaohsiung 80756, Taiwan. ${ }^{4}$ Department of Chemistry, National Taiwan Normal University, No. 88, Section 4, Ting-Chow Road, Taipei 11677, Taiwan. ${ }^{5}$ Department of Biochemical Science and Technology, National Taiwan University, No. 1, Section 4, Roosevelt Road, Taipei 10617, Taiwan. ${ }^{6}$ Institute of Bioinformatics and Structural Biology, National Tsing Hua University, 101, Kuang fu Road, Section 2, Hsinchu 30013, Taiwan. ${ }^{7}$ Department of Pathology and Laboratory Medicine, Alzheimer's Disease Center, $280550 t h$ Street, University of California Davis Medical Center, Sacramento, CA 95817, USA. ${ }^{8}$ These authors contributed equally: Yao-Hsiang Shih, Ling-Hsien Tu.

凶email: yrchen@gate.sinica.edu.tw
} 
lzheimer's disease (AD) is the most common type of dementia in which the two pathological hallmarks are senile plaques comprising amyloid- $\beta(A \beta)$ and neurofibrillary tangles composed of hyperphosphorylated tau ${ }^{1,2}$. $A \beta$, mainly consists of 40 or 42 amino acids, is a proteolytic cleavage product of amyloid precursor protein by sequential cleavages of $\beta$ - and $\gamma$-secretases. $A \beta 42$ is considered more toxic as evidenced from both in vitro ${ }^{3}$ and in vivo studies ${ }^{4}$, where $A \beta 42$ to $A \beta 40$ ratio implicated in some familial $\mathrm{AD}$ cases is also important ${ }^{5,6}$. $\mathrm{A} \beta$ spontaneously aggregates into amyloid fibrils with a cross $\beta$ sheet structure, where prefibrillar $A \beta$ oligomers are considered the most toxic species and attributed to neuronal dysfunction and cognitive impairment ${ }^{7}$. Amyloid fibrillization adopts a nucleation-dependent polymerization mechanism in which amyloid fibrils serve as a nucleus to seed soluble species and skip the nucleation ${ }^{8}$. A $\beta$ deposits extracellularly to form amyloid plaques, but also accumulate intracellularly as shown in human patients and transgenic mice ${ }^{\text {? }}$.

TDP-43 pathology was first found in cytosolic inclusions in patients with amyotrophic lateral sclerosis (ALS) and frontotemporal lobar degeneration (FTLD-TDP) ${ }^{10}$. TDP-43 inclusions were also reported in the brain of $\mathrm{AD}$ patients with a prevalence of $\sim 30$ to $\sim 57 \%^{11-13}$. The AD patients with TDP-43 inclusions have more severe memory loss and hippocampal atrophy ${ }^{13,14}$. The highly significant pathological role of TDP-43 is further suggested by a recently proposed diagnostic entity called limbicpredominant age-related TDP-43 encephalopathy (LATE), which predominantly occurs in cognitively impaired oldest-old individuals with co-existing medial temporal amyloid plaques and tauopathy ${ }^{15}$. TDP-43 is a highly conserved RNA/DNA-binding nuclear protein consisting of 414 amino acids with a molecular mass of $44,740 \mathrm{Da}^{16}$. Full-length TDP-43 is constitutively expressed in many tissues, including brain, heart, lung, kidney, and muscle ${ }^{17,18}$. TDP-43 contains two RNA binding domains (RRM1 and RRM2), which are flanked by an N-terminal domain and a $\mathrm{C}$-terminal glycine-rich region. The $\mathrm{N}$-terminal region was shown to dimerize that facilitates nucleotide binding and splicing ${ }^{19,20}$, whereas, C-terminal regions are highly prone to aggregation $^{21}$. Recombinant full-length TDP-43 forms stable spherical oligomers, which are toxic to neurons in primary culture and in mouse brain following intrahippocampal injection ${ }^{22}$. In our previous study, we generated a conformational-specific polyclonal antibody namely poly TDP-O that specifically recognizes TDP-43 oligomers and used it to confirm the existence of TDP-43 oligomers in the brain of patients with FTLD-TDP22.

Despite the common occurrence of TDP-43 proteinopathy in $\mathrm{AD}$, the pathological role of TDP-43 in $\mathrm{AD}$ is still largely unknown. A study has shown that in a transgenic AD mouse model, $3 \times$ Tg, which develops both amyloid plaques and abnormal tau, the levels of mouse TDP-43 and its C-terminal fragment were significantly increased and positively correlated with the accumulation of $A \beta$ oligomers ${ }^{23}$. In lentiviral TDP-43-injected rat, TDP-43 expression induced neuroinflammation and increased $\beta$ secretase activity, accelerating the production of A $\beta$ and APP-Cterminal fragments ${ }^{24}$. Previously, we have shown that full-length TDP-43 forms toxic spherical oligomers readily (Supplementary Fig. 1a) and can transform $A \beta$ to amyloid oligomers ${ }^{22}$. However, amyloid plaques and TDP-43 inclusions are not colocalized in the brain of $\mathrm{AD}$ patients ${ }^{25}$.

To elucidate the role of TDP-43 in $\mathrm{AD}$, here we aimed to characterize the interaction between TDP-43 and A $\beta$ and investigated the effect of TDP-43 in AD pathology. We examined the effect of TDP-43 during A $\beta$ aggregation and characterized the interaction by different TDP-43 variants. The toxicity of TDP-43induced $A \beta$ species was examined by electrophysiology and intrahippocampal injection to WT mice for memory behavior.
We further intrahippocampally injected TDP-43 in transgenic $\mathrm{AD}$ mice and showed that TDP-43 worsened the spatial memory and $\mathrm{AD}$ pathology of mice. Finally, we showed that TDP-43 oligomers mostly colocalized with intracellular $\mathrm{A} \beta$ in $\mathrm{AD}$ brain tissues.

\section{Results}

TDP-43 inhibits $A \beta$ fibrillization at the initial and oligomeric stages of $A \beta$, does not reverse the $A \beta$ fibril state, and has no impact on $\mathbf{A} \boldsymbol{\beta}$ seeding reaction. To understand the effect of TDP-43 on $A \beta$ aggregation, we first characterized $A \beta$ aggregation with and without TDP-43 by multiple biochemical techniques. A $\beta 40$ was used for all biochemical assays except for additional indication due to the relatively easy handling. First, we conducted thioflavin-T (ThT) assay to monitor the fibrillization kinetics of $\mathrm{A} \beta$ (Fig. 1a). ThT can specifically bind to cross- $\beta$-sheet structures in amyloid fibrils and emit fluorescence ${ }^{26}$. TDP-43 stock or buffer control was mixed with $A \beta$ solution, and the final $A \beta$ and TDP43 concentrations were 25 and $0.25 \mu \mathrm{M}$, respectively. The ThT results showed that $A \beta$ alone adopted a classic amyloid fibrillization pathway with a nucleation phase (lag time), an elongation phase, and a plateau phase. In the absence of TDP- $43, \mathrm{~A} \beta$ possessed a lag time of $\sim 25 \mathrm{~h}$, whereas the lag time was significantly prolonged to $\sim 75 \mathrm{~h}$ in the presence of TDP-43. The final ThT signal of A $\beta$ with TDP-43 was reduced to $~ 50 \%$ compared with the signal of $\mathrm{A} \beta$ alone. No ThT signal was found in TDP-43 alone. Next, we employed two conformation-specific antibodies, namely A11 and OC, to facilitate the understanding of the aggregation process (Fig. 1b). The time-course samples of $A \beta$ with and without TDP-43 were subjected to dot blot by using A11 and OC antibodies. A11 recognizes prefibrillar oligomers ${ }^{27}$, and OC specifically recognizes fibrillary oligomers and amyloid fibrils $^{28,29}$. At the experimental concentration, TDP-43 did not show A11 and OC signals (Supplementary Fig. 1b). During the incubation, $\mathrm{A} \beta$ alone aggregated into oligomers and gradually formed fibrils. Both A11 and OC signals were significantly enhanced at $22 \mathrm{~h}$ and saturated after $46 \mathrm{~h}$. In the presence of TDP-43, the A11 signal did not change; however, the OC signal showed delayed saturation at $70 \mathrm{~h}$ rather than $46 \mathrm{~h}$, indicating that TDP-43 delays $\mathrm{A} \beta$ fibril formation. The conformational changes in $A \beta$ were monitored at different time points through far-UV circular dichroism (CD; Fig. 1c). A $\beta$ in the absence or presence of TDP-43 was observed, and the spectra were subtracted with buffer or TDP-43 background. The CD result showed that $A \beta$ initially appeared as a random coil with a minimal signal at $198 \mathrm{~nm}$, gradually formed a $\beta$-sheet at $\sim 218 \mathrm{~nm}$ from $22 \mathrm{~h}$ to $96 \mathrm{~h}$, and saturated at $118 \mathrm{~h}$. In the presence of TDP$43, \mathrm{~A} \beta$ retained its random coil state up to $\sim 46 \mathrm{~h}$ and changed to a $\beta$-sheet structure from $70 \mathrm{~h}$ to $118 \mathrm{~h}$. This result is consistent with our observation in the ThT and dot blot assays. We observed the morphology of the aggregates through transmission electron microscopy (TEM) (Fig. 1d). At the end of aggregation ( $>160 \mathrm{~h})$, $\mathrm{A} \beta$ alone formed long, and extensive amyloid fibrils, whereas, $\mathrm{A} \beta$ in the presence of TDP-43 formed less dense and shorter filaments.

To understand how TDP-43 hinders A $\beta$ aggregation, we added TDP-43 to different $A \beta$ aggregation states and monitored the ThT signals. A $\beta$ monomers, oligomers, and preformed fibrils were prepared and validated via TEM (Supplementary Fig. 2a-c). In this set of experiments, the concentrations of $A \beta$ and TDP-43 were 25 and $0.25 \mu \mathrm{M}$, respectively. Consistent with previous results, TDP-43 added to $\mathrm{A} \beta$ monomer effectively blocked $\mathrm{A} \beta$ fibrillization (Fig. 1e). When TDP-43 was added to the A $\beta$ oligomer solution (Fig. 1f), it is still able to potently inhibit $A \beta$ fibrillization even $A \beta$ already formed oligomeric species. When 


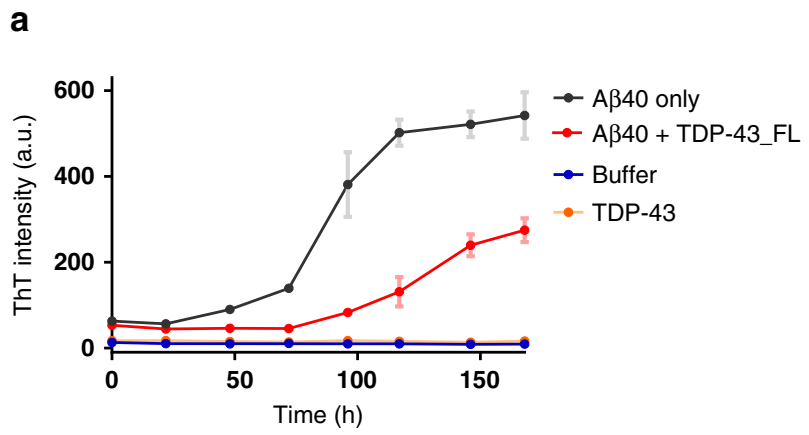

b
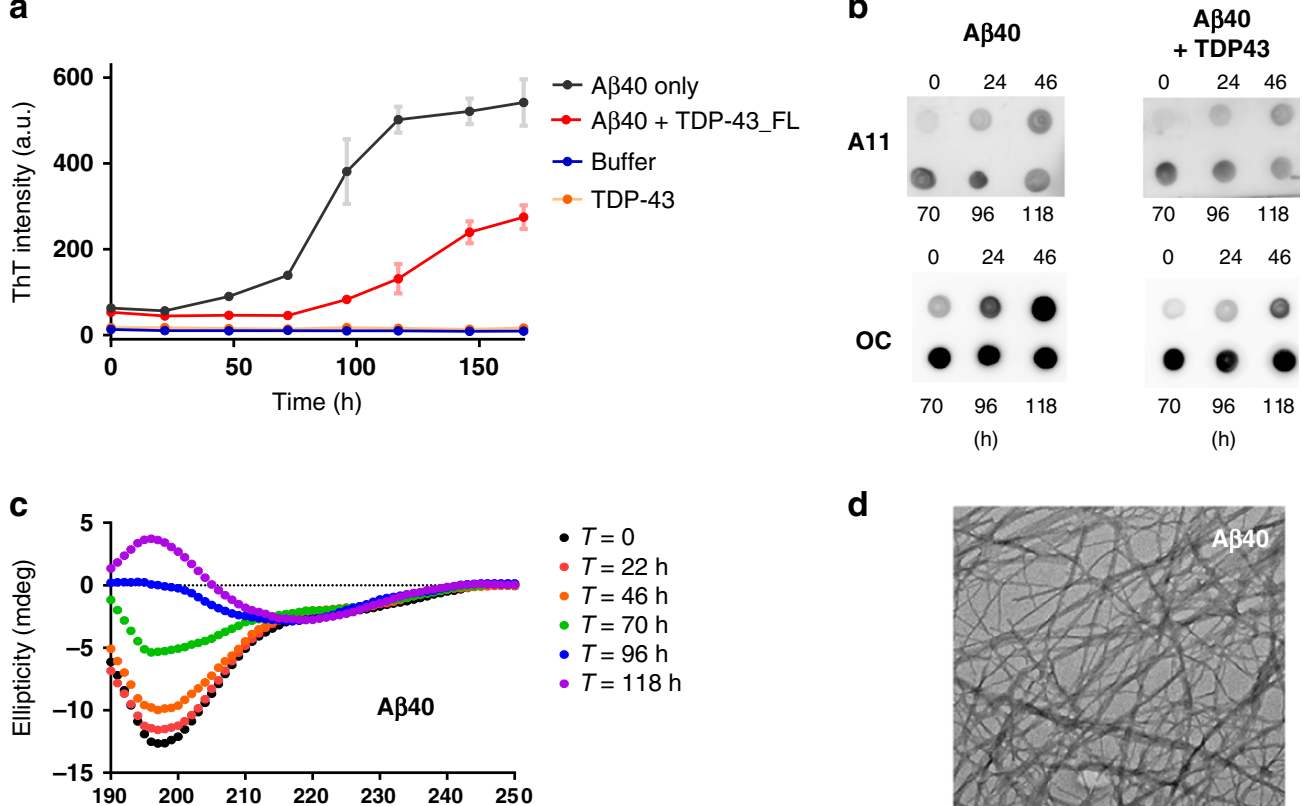

d
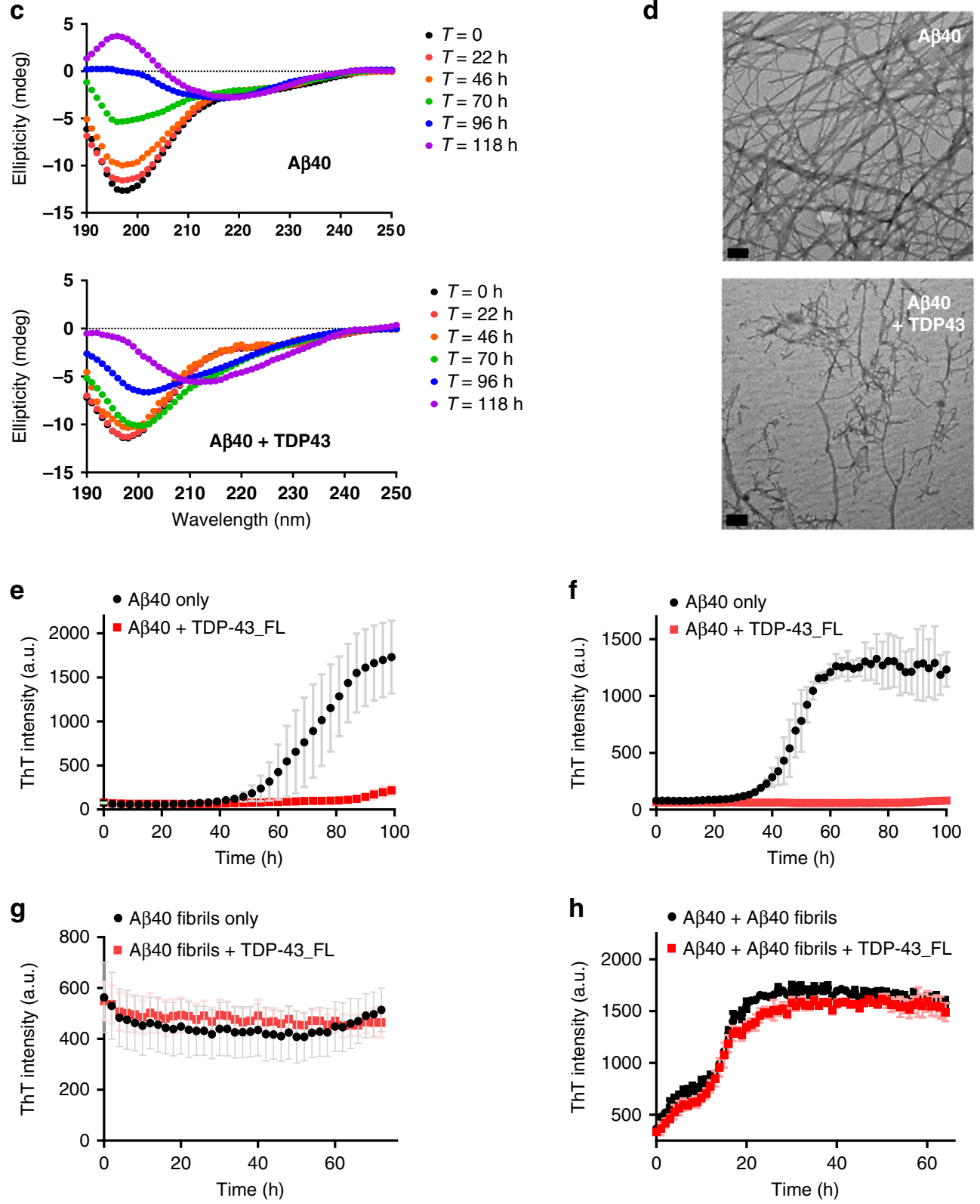

TDP-43 was introduced to the A $\beta$ fibril solution (Fig. 1g), TDP43 could not alter the status of $A \beta$ fibrils. The ThT signal of $A \beta$ fibrils remained the same during the observation. We also used immunogold TEM to observe the possible location of TDP-43 in $\mathrm{A} \beta$ fibrils by immunogold labeling of TDP-43. We found that some immunogold labeled TDP-43 attached to A $\beta$ fibrils especially at the clusters of fibrils (Supplementary Fig. 2d), but no apparent morphological changes of $\mathrm{A} \beta$ fibrils were observed. Also, we wonder if TDP-43 could block the seeding event of A $\beta$ fibrillization. $\mathrm{A} \beta$ monomer was added with $10 \%$ preformed $\mathrm{A} \beta$ fibril seeds simultaneously with and without TDP-43. The seeds were prepared from preformed $25 \mu \mathrm{M}$ A $\beta$ fibrils after sonication. The results showed that $A \beta$ fibrillization kinetics with the seeding remained the same with or without TDP-43 (Fig. 1h), indicating 


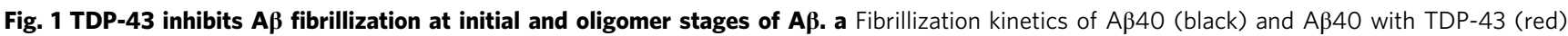
monitored by ThT assay in $10 \mathrm{mM}$ Tris buffer, pH 8.0. TDP-43 alone (orange) and buffer alone (blue) are also shown. The averaged data from three replicates and standard deviations are plotted. A 340 concentration was $25 \mu \mathrm{M}$ and TDP-43 concentration was $0.25 \mu \mathrm{M}$. b Dot blotting of the time point samples by anti-amyloid oligomers A11 antibody and anti-amyloid fibrils OC antibody. c Far-UV CD spectra of A 340 in the absence and presence of TDP43_FL after 0 (black), 22 (red), 46 (orange), 70 (green), 96 (blue), and 118 (purple) h incubation. The buffer control and TDP-43 background were subtracted. d TEM images of the end-point product of $A \beta 40$ alone in ThT assay, and the end-point product of A $\beta 40$ with TDP-43. The scale bars are 100 $\mathrm{nm}$. Two times of independent experiments were performed. e-h Fibrillization kinetics of A 340 from different stages with and without full-length TDP-43. $A \beta 40$ monomer (e), $A \beta 40$ oligomers (f), $A \beta 40$ fibrils (g), and $A \beta 40$ monomer in the presence of $10 \%$ fibril seeds (h) were incubated with (red) and without full-length TDP-43 (black) and monitored by ThT assay in $10 \mathrm{mM}$ Tris buffer, pH 8.0. A 440 concentration was $25 \mu \mathrm{M}$, and TDP-43 concentration was $0.25 \mu \mathrm{M}$. A $\beta$ fibril seeds were prepared from sonicated $A \beta 40$ fibrils at $25 \mu \mathrm{M}$. The averaged data from three replicates and standard deviations are plotted. Source data are provided as a Source Data file.

that TDP-43 could not prevent A $\beta$ fibril growth by seeding. Therefore, our result showed that TDP-43 could only inhibit A $\beta$ fibrillization at the initial and oligomeric states, but not affect $A \beta$ conformation at the fibril state and in the seeding reaction.

$\mathrm{N}$-terminal containing TDP-43 variants inhibit A $\beta$ fibrillization. TDP-43 contains four structural regions, which are an $\mathrm{N}$-terminal domain, two RNA recognition motifs (RRM1 and RRM2), and a C-terminal glycine-rich region (Fig. 2a). TDP-43 may function as a homodimer via the interaction of $\mathrm{N}$-terminal regions $^{20,30}$, but the misfolded structure of TDP-43 has not been clearly elucidated. In our previous study, full-length TDP-43 readily formed oligomers that were eluted in the void volume in size exclusion chromatography (SEC) ${ }^{22}$. Here, we aimed to clarify the prerequisite structural regions of TDP-43 for inhibiting A $\beta$ fibrillization. We constructed and purified different structural regions of TDP-43, including TDP-43 with a C-terminal region truncated (named TDP-43_265, residue 1-265), an N-terminal region (named TDP-43_N-term, residue 1-100), and the RNA recognition region (named TDP-43_RRM1 + 2, residue 101-265). The calculated molecular weights of each variant were $32.5 \mathrm{kDa}$ for TDP-43_265, $12.5 \mathrm{kDa}$ for TDP-43_N-term, and $20.4 \mathrm{kDa}$ for TDP-43_RRM1 + 2. The assembly of these truncated proteins was first characterized in SEC (Supplementary Fig. 3a). According to the molecular weight standards, TDP-43_265 and TDP-43_Nterm formed homodimers with estimated molecular masses of 53 and $23 \mathrm{kDa}$, but TDP-43_RRM1 +2 remained monomeric with an estimated molecular mass of $21 \mathrm{kDa}$. No aggregates in these TDP-43 variants were observed in SEC. We subjected the variants to dot blot and found that only full-length TDP-43 was immunopositive and that TDP-43_265 was very weakly immunopositive when probed by our polyclonal TDP-43 oligomer-specific antibody TDP-O 22 (Supplementary Fig. 3b).

Next, we tested the potency of TDP-43 variants in inhibiting A $\beta$ fibrillization (Fig. 2b). As expected, full-length TDP-43 inhibited the formation of $\mathrm{A} \beta$ fibrils by prolonging the lag time and lowering the ThT fluorescence to $60 \%$ at the final time point. Surprisingly, TDP-43_265 and TDP-43_N-term exhibited strong inhibition on A $\beta$ fibrillization. TDP-43_N-term prolonged the lag time of $\mathrm{A} \beta$ from $50 \mathrm{~h}$ to $\sim 90 \mathrm{~h}$ and reduced ThT intensity to $75 \%$ compared with that of the A $\beta$ only control, while TDP-43_265 prolonged the lag time from $50 \mathrm{~h}$ to $125 \mathrm{~h}$, and reduced the ThT signal to $\sim 9 \%$. By contrast, TDP-43_RRM1 +2 did not have much inhibitory effect on $A \beta$ fibrillization, with the lag time of $A \beta$ slightly prolonged from $50 \mathrm{~h}$ to $55 \mathrm{~h}$, and the final ThT intensity slightly affected (decreased to $\sim 90 \%$ ). We employed TEM to observe the morphology of $\mathrm{A} \beta$ species at 100 and $150 \mathrm{~h}$ incubation (Fig. 2c). In the absence of TDP-43 variants, $A \beta$ formed typical amyloid fibrils through time as expected. However, A $\beta$ samples with full-length TDP-43, TDP-43_265, or TDP-43_N-term contained much less and shorter fibrils than A $\beta$ alone, whereas, A $\beta$ with TDP-43_RRM1 +2 still contained a significant number of fibrils.

The end-products of aggregation study were further examined by western blot (Fig. $2 \mathrm{~d}, \mathrm{e}$ ). The samples collected after the endpoint $(150 \mathrm{~h})$ of the $\mathrm{ThT}$ assay were stored at $-20^{\circ} \mathrm{C}$ and later subjected to sodium dodecyl sulfate polyacrylamide gel electrophoresis (SDS-PAGE). The samples were loaded onto TrisTricine SDS-PAGE and transferred onto a polyvinylidene fluoride (PVDF) membrane probed by the anti-A $\beta$ antibody $6 \mathrm{E} 10$. We found that $\mathrm{A} \beta$ alone possessed a large amount of aggregates stocked on top of the gel (lane 5, Fig. $2 \mathrm{~d}$ ) so as $A \beta$ with TDP-43 RRM1 + 2 (lane 4). A $\beta$ incubated with full-length TDP43 had few $A \beta$ aggregates on top of the gel (lane 1). A $\beta$ with TDP43_265 and TDP-43_N-term (lane 2 and 3) had reduced aggregates compared with $\mathrm{A} \beta$ only. Considering that the aggregates stocked on top of the gel were not quantitative, we examined the lower portion of the SDA-PAGE. After enhancing the contrast of the low-molecular-weight species (Fig. 2e), we found that $A \beta$ in the presence of full-length TDP-43 significantly enhanced $A \beta$ monomer, dimer, and trimer (lane 1 ). $A \beta$ in the presence of TDP-43_265 and TDP-43_N-term (lane 2 and 3) also showed a significant enhancement of monomer and dimer compared with A $\beta$ alone (lane 5) and A $\beta$ with TDP43_RRM1 +2 (lane 4). Overall, the results demonstrated that full-length, residues 1-265, and N-terminal TDP-43 could inhibit A $\beta$ fibrillization, leading to fibril reduction and low-molecularweight species accumulation. We also tested the inhibitory effect of TDP-43 variants on A $\beta 42$ fibrillization by ThT assay. The ThT result showed that full-length TDP-43 still significantly inhibited A $\beta 42$ fibrillization by retarding the lag time from $20 \mathrm{~h}$ to $40 \mathrm{~h}$ (Supplementary Fig. 4). However, the other TDP-43 variants did not significantly affect $A \beta 42$ fibrillization.

TDP-43 variants interact with $\mathrm{A} \beta$ via different structural domains. Considering that TDP-43 variants inhibited $\mathrm{A} \beta$ aggregation to different degrees, we examined the molecular interaction between TDP-43 variants and $A \beta$. We first employed enzyme-linked immunosorbent assay (ELISA) to detect the binding of $A \beta$ to TDP-43 proteins (Fig. 3a). In this experiment, TDP-43 variants at $1 \mu \mathrm{M}, 200$ pmole, were immobilized onto the ELISA plate. N-terminal biotinylated $\mathrm{A} \beta 40$ was added at various concentrations ranging from 0 to $38 \mu \mathrm{M}$ and subjected to streptavidin- horseradish peroxidase (HRP) detection. The ELISA results showed that the binding of $A \beta$ was the strongest in the full-length TDP-43, followed by TDP-43_265 and TDP43_RRM1 +2 . The weakest binding was found in the N-terminal TDP-43.

We further performed biolayer interferometry analysis to examine the on and off kinetic binding rates of TDP-43 variants (Fig. 3b-d). The biotinylated $\mathrm{A} \beta 40$ was immobilized onto streptavidin-coated sensors and then subjected to different concentrations of TDP-43 variants to detect the interaction 
a

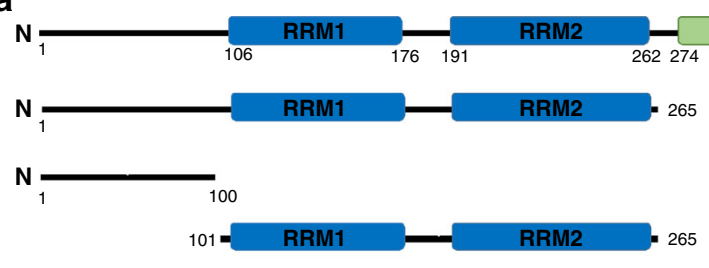

TDP-43_FL

TDP-43_265

TDP-43 $\mathrm{N}$ term

TDP-43 RRM1 + 2

RNA recognition motifs

b

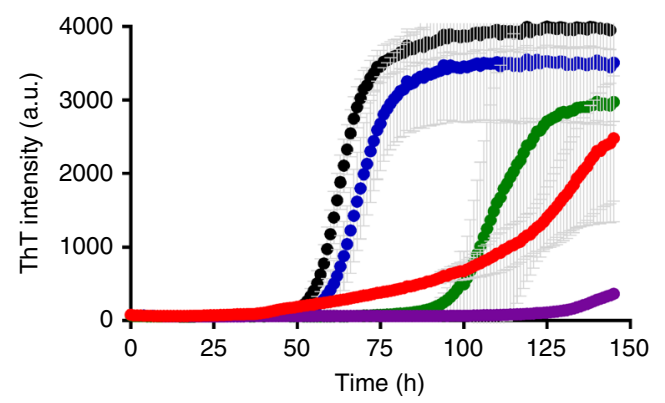

Glycine-rich region

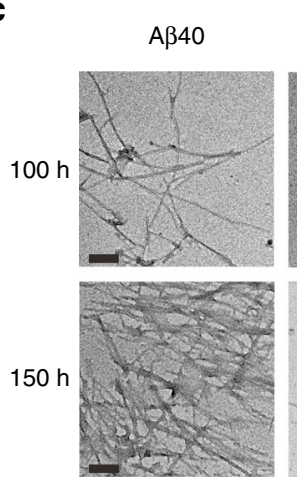

d
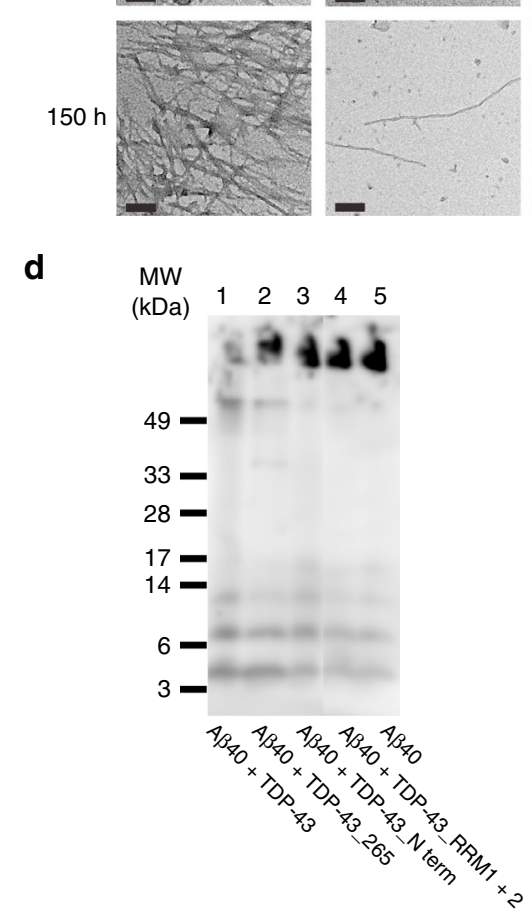

- $A \beta 40$

- $A \beta 40+$ TDP-43

- AB40 + TDP-43_265

- $A \beta 40+$ TDP-43_N term

- $A \beta 40$ + TDP-43_RRM1 + 2
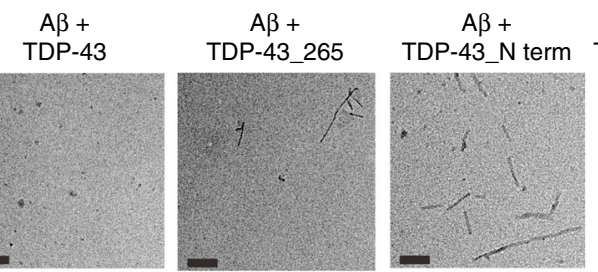

$A \beta+$
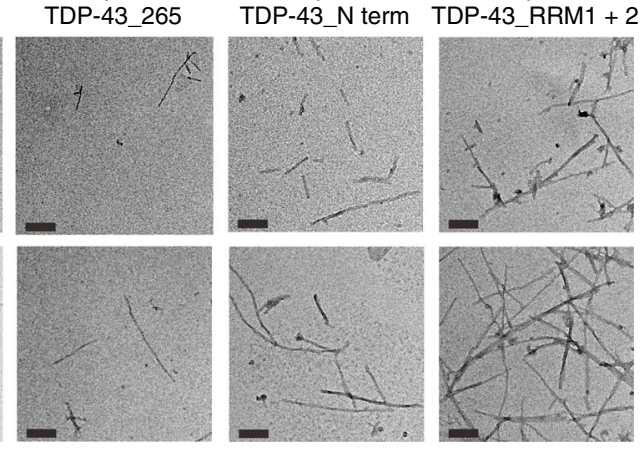

e

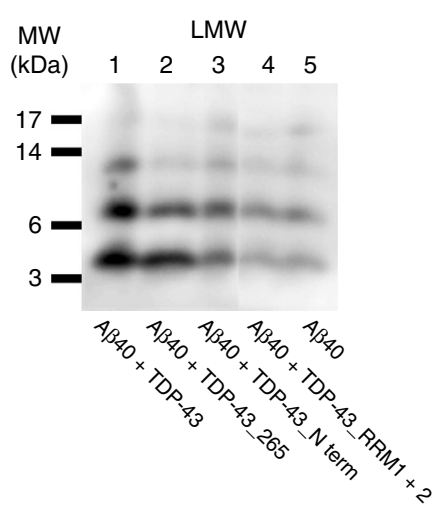

Fig. 2 Truncated TDP-43 variants inhibit A $\beta$ fibrillization. a Illustration of the structural motifs of TDP-43. Full-length TDP-43 contains an N-terminal domain, two RNA recognition motifs (RRM1 and RRM2), and a glycine-rich region in its C-terminus. The constructs used in the study are shown. They are full-length TDP-43, TDP-43 aa 1-265 (TDP-43_265), TDP-43 aa 101-265 (TDP-43_RRM1 + 2), and TDP-43 aa 1-100 (TDP-43_N-term). b ThT assays of A $\beta$ fibrillization (black) and with TDP-43 variants (TDP-43_FL, red; TDP-43_265, purple; TDP-43_N-term, green; TDP-43_RRM1 + 2, blue) in 10 mM Tris buffer, $\mathrm{pH}$ 8.0. A $\beta 40$ concentration was $25 \mu \mathrm{M}$, and TDP-43 concentration was $0.25 \mu \mathrm{M}$. The averaged data from three replicates and standard deviations are plotted. c TEM images of A $\beta$ species with and without TDP-43 proteins. Two incubation time points, $100 \mathrm{~h}$ and $150 \mathrm{~h}$ were chosen for examination. The samples were loaded on SDS-PAGE $\mathbf{d}$ and then probed by anti-A $\beta$ antibody, 6 E10 to show the relative amounts of A $\beta 40$ amyloid fibrils. Two times of independent experiments were performed. $\mathbf{e}$ The enlarged area of Fig. $2 \mathrm{~d}$ shows a clear band distribution of low-molecular-weight $A \beta$ species. Source data are provided as a Source Data file. 
a

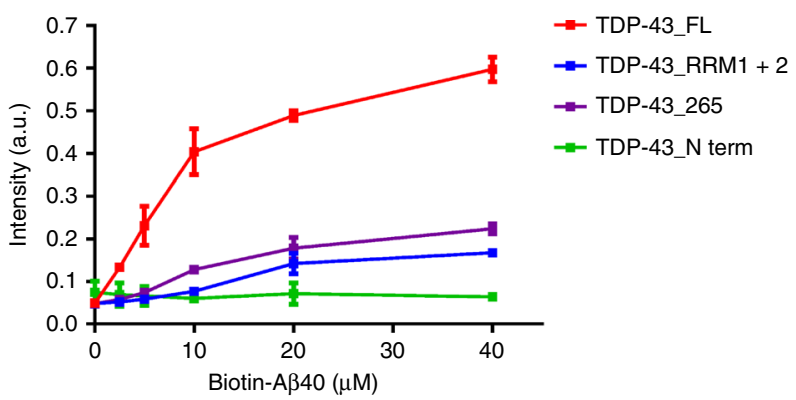

b

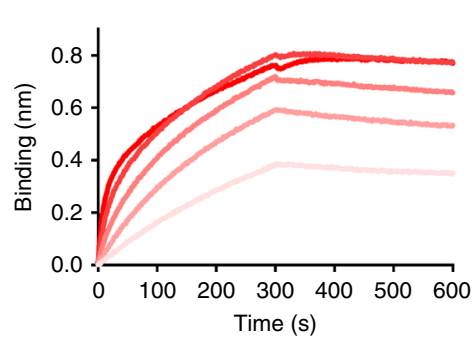

d

TDP-43_N term

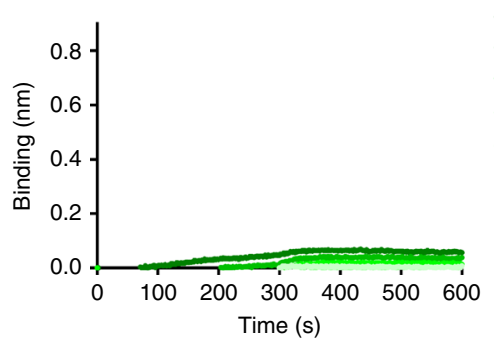

C

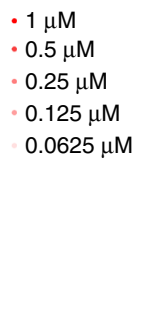

$3 \mu \mathrm{M}$

- $1.5 \mu \mathrm{M}$

$0.75 \mu \mathrm{M}$

$0.375 \mu \mathrm{M}$

$0.1875 \mu \mathrm{M}$

e
- $3 \mu \mathrm{M}$

- $1.5 \mu \mathrm{M}$

$\cdot 0.75 \mu \mathrm{M}$

$0.375 \mu \mathrm{M}$

$0.1875 \mu \mathrm{M}$

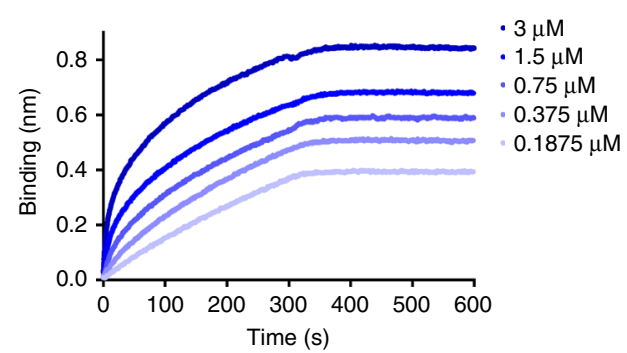

Fig. 3 Interaction of TDP-43 variants and A $\boldsymbol{\beta}$. a Interaction of A $\beta$ with TDP-43 variants was determined by ELISA. TDP-43 proteins were coated and detected by different concentrations of biotinylated A $\beta$. The averaged data from three replicates and standard deviations are plotted (TDP-43_FL, red; TDP43_265, purple; TDP-43_N-term, green; TDP-43_RRM1 + 2, blue). b-e Binding response between A 340 and TDP-43 variants in biolayer interferometry analysis. TDP-43 at different concentrations $(\mu \mathrm{M})$ were prepared and subjected to biolayer interferometry analysis by using Octet RED96 System (Pall ForeBio). The real-time binding response ( $\mathrm{nm}$ ) was measured in seconds. The loading amounts of A $\beta 40$ are the same for all the experiments. Source data are provided as a Source Data file.

Table 1 Affinity of TDP-43 variants and A $\beta$ interaction. The rate constants $\boldsymbol{k}_{\text {on }} \boldsymbol{k}_{\text {off, }}$ and $\boldsymbol{K}_{\mathrm{D}}$ defined as $\boldsymbol{k}_{\text {off }} / \boldsymbol{k}_{\text {on }}$ were determined by Octet RED96 system to probe the interaction between TDP-43 and A $\beta 40$.

\begin{tabular}{llll} 
& $\boldsymbol{k}_{\text {on }}(\mathbf{1} / \mathbf{M s})$ & $\boldsymbol{k}_{\text {off }}(\mathbf{1} / \mathbf{s})$ & $\boldsymbol{K}_{\mathbf{D}} \mathbf{( M )}$ \\
\hline TDP-43_FL & $3.4 \times 10^{4}$ & $<1.0 \times 10^{-7}$ & $<1.0 \times 10^{-12}$ \\
TDP-43_265 & $5.7 \times 10^{3}$ & $5.6 \times 10^{-4}$ & $9.9 \times 10^{-8}$ \\
TDP-43_N-term & N.D. & N.D. & N.D. \\
TDP-43_RRM1 + 2 & $9.0 \times 10^{3}$ & $<1.0 \times 10^{-7}$ & $<1.0 \times 10^{-12}$ \\
\hline N.D.: unable to be determined due to absence of significant binding signal. & \\
\hline
\end{tabular}

between $A \beta$ and TDP-43. Owing to the strong nonspecific binding of TDP-43_N-term to streptavidin-coated sensors, we added $0.005 \%$ Tween-20 in all experiments to reduce nonspecific binding of TDP-43. The sensograms were plotted, and $k_{\text {on }}, k_{\text {off, }}$, and $K_{\mathrm{D}}$ were calculated (Table 1). The parameters were successfully obtained for all TDP-43 variants except TDP-43 Nterm. The $k_{\text {on }}$ rates for all other variants are around $10^{3}-10^{4} \mathrm{M} / \mathrm{s}$. Both full-length TDP-43 and TDP-43_RRM1 +2 have $k_{\text {off }}$ rate $<10^{-7} / \mathrm{s}$ indicating tight binding without easy dissociation. The small $k_{\text {off }}$ rate resulted in a small dissociation constant for both TDP-43_FL and TDP-43_RRM1 $+2\left(K_{\mathrm{D}}<10^{-12} \mathrm{M}\right)$. For TDP$43 \_265$, the dissociation constant is $9.87 \times 10^{-8} \mathrm{M}$.

TDP-43-induced A $\beta$ species impair long-term-potentiation (LTP) in the hippocampus and the related spatial memory in vivo. $L T P$ is an important cellular mechanism related to learning and memory function ${ }^{31}$. To investigate whether TDP43 -induced changes in $\mathrm{A} \beta 40$ or $\mathrm{A} \beta 42$ aggregation impaired neuronal function, we measured Schaffer collateral-CA1 LTP in mouse brain slice (Fig. 4) after treatment of the aggregation samples as described in Fig. 1a. The brain slices were incubated with TDP-43-induced $A \beta$ species, $A \beta$ alone, TDP-43 alone, and buffer control for $30 \mathrm{~min}$, and the LTP responses were measured after a theta burst stimulation. The A $\beta 40$ experiments (Fig. 4a) showed that TDP-43-induced A $\beta 40$ species significantly suppressed hippocampal LTP compared with that of the buffer control (repeated two-way ANOVA, treatment factor: $F=28.21$, df $1 / 5, p=0.003$ ). However, no significant difference in the fEPSP slope was induced by the buffer control, A $\beta 40$ alone $(1 \mu \mathrm{M})$, and TDP-43 alone $(0.01 \mu \mathrm{M})$ (repeated two-way ANOVA, treatment factor: $F=1.21$, df $2 / 8, p=0.348$ ). In $\mathrm{A} \beta 42$ experiments, we prepared $A \beta 42$ following previous literature ${ }^{32}$ and incubated with 
a

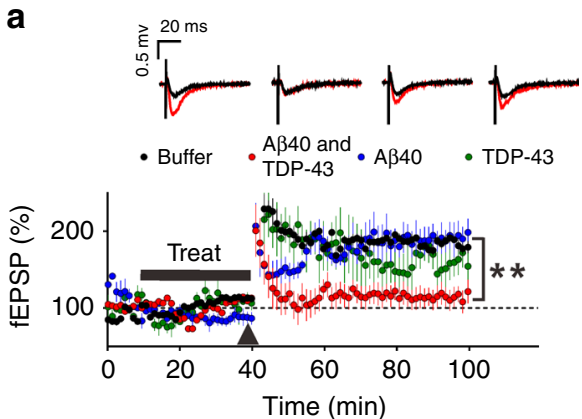

b

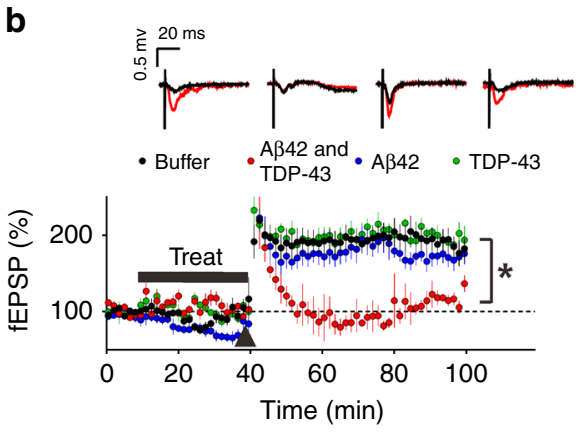

Fig. 4 TDP-43-induced A $\boldsymbol{\beta}$ species impairs the hippocampus LTP. Field EPSPs (fEPSPs) were measured from a Schaffer collateral fiber on a hippocampal slice from C57BL/ 6 mice. After the recording was stabilized for 10 min, the slices were treated with buffer, A $\beta$ with TDP-43, A $\beta$ alone, or TDP-43 alone for $30 \mathrm{~min}$. After the treatment, the hippocampal slices were subjected to a theta burst stimulation (black arrowhead) to induce LTP. Scale bar, $0.5 \mathrm{mv}, 20 \mathrm{~ms}$. The averaged data and s.e.m. are plotted and colored for buffer control (black), A $\beta$ and TDP-43 (red), A $\beta$ (blue), and TDP-43 (green). Each group of fEPSPs before (black line) and after (red line) the theta burst stimulation is shown individually in the upper panel. a For A $\beta 40$ analysis, A $\beta 40$ at $1 \mu M$ and TDP-43 at $10 \mathrm{nM}$ were used. The slices were treated with buffer $(n=4$ independent slices), A $\beta 40$ with TDP-43 $(n=3$, independent slices), A $\beta 40$ alone ( $n=4$, independent slices), or TDP-43 alone ( $n=4$, independent slices). TDP-43 + A $\beta 40$ vs. buffer; repeated two-way ANOVA, $p=0.0032, * * p<0.01$. b For A $\beta 42$ analysis, $A \beta 42$ at $62.5 \mathrm{nM}$ and TDP-43 at $0.625 \mathrm{nM}$ were used. The slices were treated with buffer $(n=9$ independent slices), A 342 with TDP-43 ( $n=4$, independent slices), A $\beta 42$ alone ( $n=5$, independent slices), or TDP-43 alone ( $n=5$, independent slices). TDP- $43+$ A $\beta 42$ vs. buffer; repeated twoway ANOVA, $p=0.0232,{ }^{*} p<0.05$. Source data are provided as a Source Data file.

a
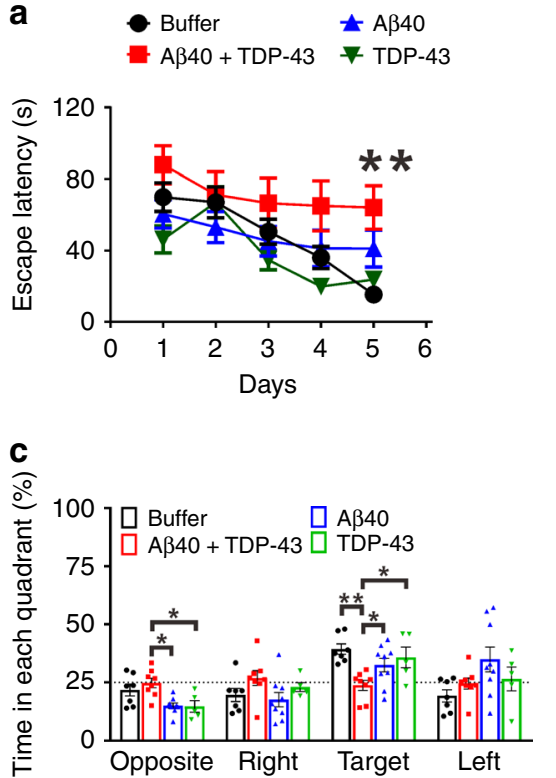

b
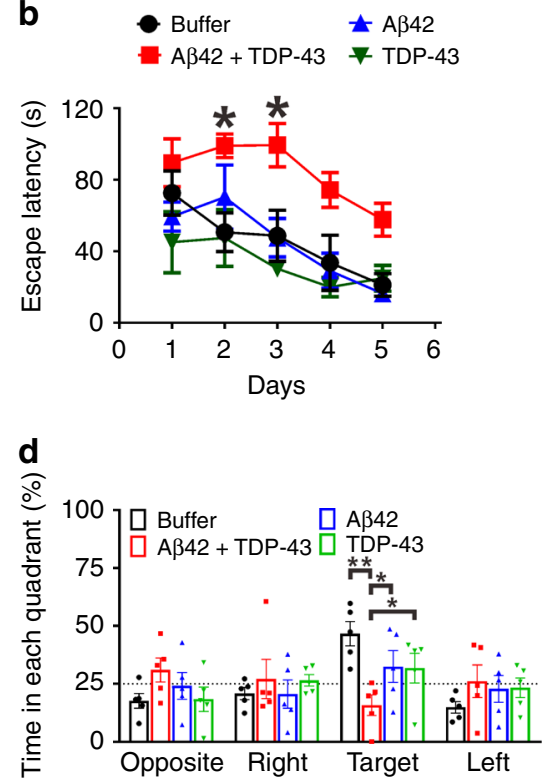

Fig. 5 TDP-43-induced A $\beta$ species impairs hippocampus-related spatial memory to a greater degree than A $\beta$ or TDP-43 alone does in the injected mouse model. WT mice at the age of 5 -month were intracranially injected with the samples from the aggregation assays. A $\beta$, TDP-43, A $\beta$ with TDP-43, and buffer obtained from the aggregation reaction were used. Spatial learning and memory functions were inspected by MWM 1 month after injection. Escape latency is defined as the time at which the hidden platform is found. The data were colored for buffer control (black), A $\beta$ and TDP-43 (red), A $\beta$ (blue), and TDP-43 (green). a Training phase for A $\beta 40$. The sample sizes for buffer, A $\beta 40$ with TDP-43, A $\beta 40$, and TDP-43 are 7, 8, 9, and 5, respectively. The averaged data and s.e.m. are plotted. The statistical analysis was performed by repeated two-way ANOVA and Bonferroni's post-hoc test, ${ }^{\star \star} p<0.01$ (Buffer vs. A $\beta 40+$ TDP-43 at day 5, $p=0.0048$ ). b Training phase for A $\beta 42$. The sample size for buffer, A $\beta 42$ with TDP-43, A $\beta 42$, and TDP-43 are 5, 4, 4 , and 5, respectively. The averaged data and s.e.m. are plotted. The statistical analysis was performed by repeated two-way ANOVA and Bonferroni's post-hoc test, ${ }^{\star} p<0.05$ (Buffer vs. A $\beta 42+$ TDP-43 at day 2, $p=0.0174 ;$ Buffer vs. A $\beta 42+$ TDP-43 at day 3, $p=0.0112$ ). c Probe test for A $\beta 40$. The time in each quadrant were calculated in percentage. The averaged data and s.e.m. are plotted. The statistical analysis was performed by one-way ANOVA, Holm-Sidak's multiple comparisons, ${ }^{\star} p<0.05,{ }^{\star \star} p<0.01$ (In the opposite quadrant: A $\beta 40+$ TDP-43 vs. A $\beta 40, p=0.0147 ;$ A $\beta 40+$ TDP-43 vs. TDP-43, $p=0.038$; In the target quadrant, Buffer vs. $A \beta 40+$ TDP-43, $p=0.002 ; A \beta 40+$ TDP-43 vs. A $\beta 40, p=0.0425 ; A \beta 40+$ TDP-43 vs. TDP-43, $p=$ 0.0289). d Probe test for A $\beta 42$. The averaged data and s.e.m. are plotted. The statistical analysis was performed by one-way ANOVA, Holm-Sidak's multiple comparisons, ${ }^{\star} p<0.05,{ }^{\star \star} p<0.01$ (In the target quadrant: Buffer vs. A $\beta 42+$ TDP-43, $p=0.0019 ; A \beta 42+$ TDP-43 vs. A $\beta 42, p=0.0311 ; A \beta 42+$ TDP-43 vs. TDP-43, $p=0.0311$ ). Source data are provided as a Source Data file. 


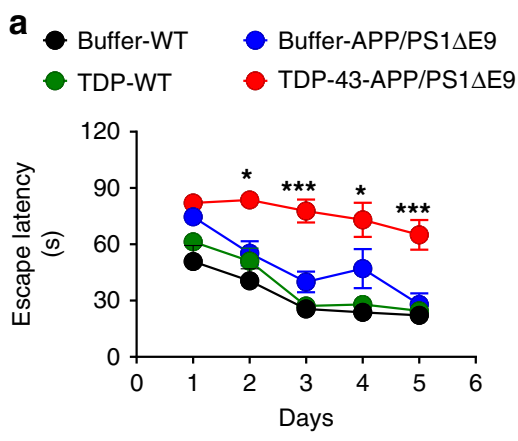

C
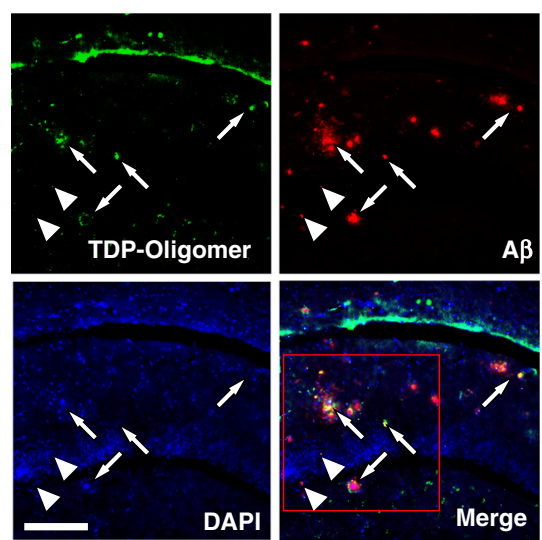

e
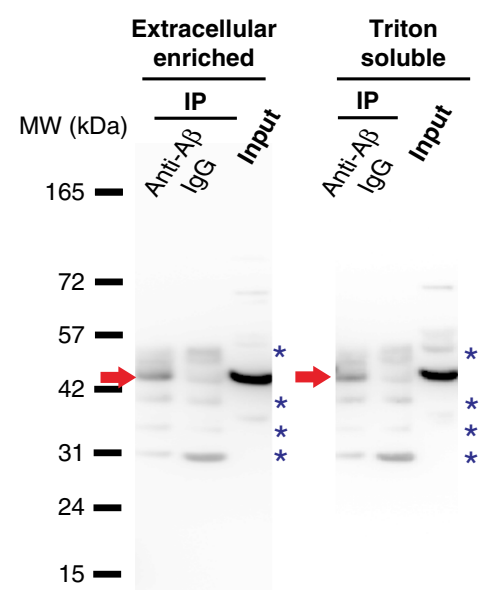

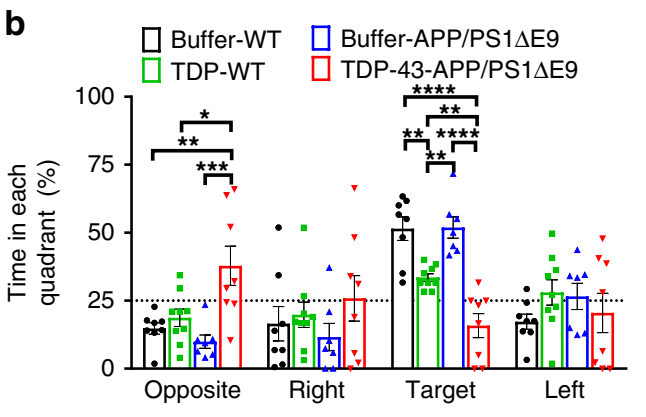

d
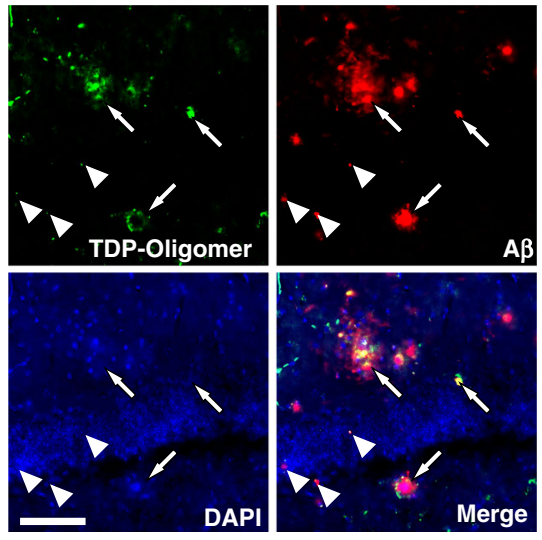

f

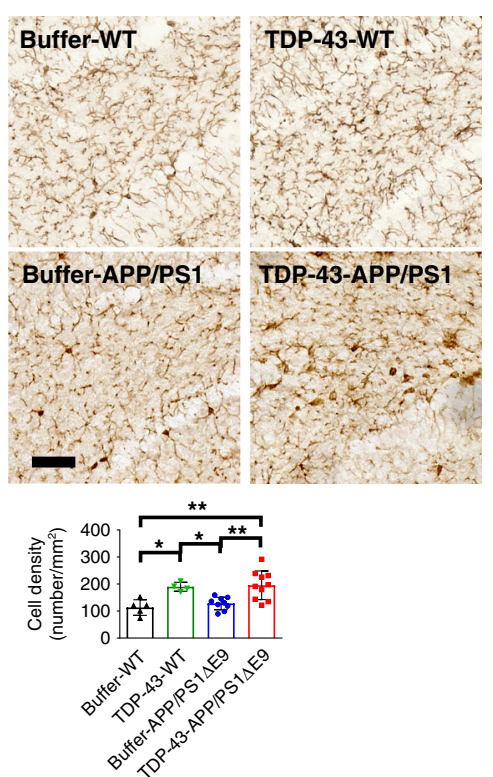

in the fEPSP slope among the buffer control, $62.5 \mathrm{nM} \mathrm{A} \beta 42$, and $0.625 \mathrm{nM}$ TDP-43 (repeated two-way ANOVA, treatment factor: $F=0.5092$, df $2 / 15, p=0.611$ ). The result indicated that TDP-43induced $A \beta$ species significantly impair synapse function to a greater extent than $\mathrm{A} \beta$ alone or TDP-43 alone did.

Next, to investigate the detrimental effect of TDP-43-induced $\mathrm{A} \beta$ species on the hippocampus-related spatial learning and memory in vivo, we injected the aggregation samples to the hippocampi of wild-type mice and examined the mouse behavior by Morris water maze (MWM) paradigm 1 month after the injection (Fig. 5). Two microliters of the recombinant $\mathrm{A} \beta 40$ or 42 $(25 \mu \mathrm{M})$, full-length TDP-43 $(0.25 \mu \mathrm{M})$, TDP-43-induced $\mathrm{A} \beta$ (25 $\mu \mathrm{M}$ A $\beta 40$ or $42,0.25 \mu \mathrm{M}$ TDP-43), or buffer control obtained from the aggregation assay were injected. In the pre-training 
Fig. 6 TDP-43 impairs spatial memory, colocalizes and interacts with A $\beta$, and increases microgliosis in APP/PS1 $\Delta$ E9 mice. a, b The spatial learning and memory function of APP/PS1 $\triangle$ E9 mice were inspected via MWM. The training phase (a) and probe test (b) are shown (buffer, $n=6$, TDP-43, $n=8)$. a For the training phase, the averaged data and s.e.m. are plotted. The data were colored for buffer-injected WT mice (black), TDP-43-injected WT mice (green), buffer-injected APP/PS1 $\triangle$ E9 mice (blue), and TDP-43-injected APP/PS1 $\triangle$ E9 mice (red). Statistical analysis was performed via repeated two-way ANOVA with Bonferroni's post-hoc test, ${ }^{\star} p<0.05,{ }^{\star \star \star} p<0.001$ (Buffer-APP/PS1 $\Delta$ E9 vs. TDP-43-APP/PS1 $\Delta$ E9 at day $2, p=0.0190 ;$ at day 3, $p=0.0002 ;$ at day 4 , $p=0.0135$; at day 5, $p=0.0002$ ). b For the probe test, the average data and s.e.m. are plotted. Statistical analysis was conducted with one-way ANOVA, Holm-Sidak's multiple comparisons, ${ }^{\star} p<0.05,{ }^{\star \star} p<0.01,{ }^{\star \star \star} p<0.001,{ }^{\star \star \star \star} p<0.0001$ (In the opposite quadrant, Buffer-WT vs. TDP-43-APP/PS1 $\Delta$ E9, $p=$ 0.0036; TDP-WT vs. TDP-43-APP/PS1 $\Delta E 9, p=0.0122 ;$ Buffer-APP/PS1 $\Delta$ E9 vs. TDP-43-APP/PS1 $\Delta E 9, p=0.0008$; In the target quadrant: Buffer-WT vs. TDP-43-WT, $p=0.0046$; Buffer-APP/PS1 $\Delta$ E9 vs. TDP-43-APP/PS1 $\Delta$ E9, $p<0.0001 ;$ TDP-WT vs. TDP-43-APP/PS1 $\Delta$ E9, $p=0.0046 ;$ buffer-WT vs. TDP43-APP/PS1 $\Delta$ E9, $p<0.0001$; TDP-WT vs. buffer-APP/PS1 1 E9, $p=0.0046)$. c Representative immunostaining micrographs in the hippocampus dentate gyrus show that TDP-43 oligomers colocalized with A $\beta$ plaque (arrows) and intraneuronal A $\beta$ (arrowhead; scale bar, $150 \mu \mathrm{m}$ ). Three induvial animals in each group were examined. $\mathbf{d}$ The enlarged view of the rectangle in $6 \mathrm{c}$ (scale bar, $100 \mu \mathrm{m}$ ). e Representative IP result of A $\beta$ and TDP-43 in the brain fractions of APP/PS1 $\triangle E 9$ mice injected with TDP-43. Extracellular-enriched and Triton-soluble brain fractions were used. IP was performed using A $\beta$ antibodies and detected by TDP-43 antibodies. Immunoprecipitated TDP-43 was indicated by red arrows and nonspecific bands were indicated by blue asterisks. Four independent experiments were performed. f TDP-43 injection increased microgliosis in the hippocampus dentate gyrus of APP/PS1 $\triangle$ E9 mice. Representative lba1 immunostaining micrographs of buffer-injected $(n=5)$ or TDP-43-injected $(n=4)$ wild-type mice and buffer-injected ( $n=9)$ or TDP-43-injected $(n=10)$ APP/PS1 $\Delta$ E9 mice. The calculated cell density of lba1-positive microglial cell is shown (scale bar, $30 \mu \mathrm{m})$. The averaged data and s.e.m. are plotted. Statistical analysis was performed by two-tailed Mann-Whitney test, ${ }^{\star} p<0.05,{ }^{\star \star} p<0.01$ (Buffer-WT vs. TDP-43-WT, $p=0.0159$; Buffer-WT vs. TDP-43-APP/PS1 $\Delta$ E9, $p=0.0043$; TDP-43-WT vs. Buffer-APP/PS1 $\Delta$ E9, $p=0.0238 ;$ Buffer-APP/PS1 $\Delta$ E9 vs.TDP-43-APP/PS1 $\Delta$ E9, $p=$ 0.0076). Source data are provided as a Source Data file.

phase, all the groups showed a similar trend in a time-dependent decrease in escape latency demonstrating no sample injection effect (Supplementary Fig. 6a, b) (for A $\beta 40$ study, repeated twoway ANOVA, time factor: $F=54.47$; $\mathrm{df} 1 / 26 ; p<0.001$; treatment factor, $F=2.875$; df $3 / 26 ; p=0.055$; for $\mathrm{A} \beta 42$ study, time factor: $F=25.60$, df $1 / 15, p=0.0001$, treatment factor: $F=0.82$, df $3 / 15$, $p=0.505)$. The swimming velocity for each group was unchanged (Supplementary Fig. 6c, d) (for A $\beta 40$ study, one-way ANOVA, $F=0.016$, df $3 / 26, p=0.997$; for A $\beta 42$ study, $F=0.163$, df $3 / 16$, $p=0.354)$.

In the training phase, the escape latency time of the TDP-43induced $A \beta 40$ (Fig. 5a) or $A \beta 42$ (Fig. 5b) group was significantly increased compared with that of the buffer group (for A $\beta 40$ study, repeated two-way ANOVA, $F=5.25$, df $1 / 13, p=$ 0.039 ; for $\mathrm{A} \beta 42$ study, $F=11.07$, df $1 / 8, p=0.010$ ). More importantly, the escape latency time of the TDP-43-induced A $\beta$ was significantly increased compared with that of $A \beta$ alone (for A $\beta 40$ group, repeated two-way ANOVA, $F=8.385$, df $1 / 14, p=$ 0.012 ; for $\mathrm{A} \beta 42$ group, $F=19.14$, df $1 / 7, p=0.003)$ and TDP-43 alone (for $A \beta 40$ group, repeated two-way ANOVA, $F=6.451$, df $1 / 11, p=0.0275$; for $\mathrm{A} \beta 42$ group, $F=34.73$, df $1 / 8, p=0.0004$ ). In the probe test with the platform removed, the mice injected with TDP-43-induced A $\beta 40$ (Fig. 5c) or A $\beta 42$ (Fig. 5d) spent significantly less time than the other groups in the target quadrant (for A 340 study, one-way ANOVA, Holm-Sidak's multiple comparisons, $\mathrm{A} \beta+\mathrm{TDP}$ vs. buffer, $p=0.002 ; \mathrm{A} \beta+\mathrm{TDP}$ vs. $\mathrm{A} \beta, \quad p=0.0425 ; \mathrm{A} \beta+\mathrm{TDP}$ vs. TDP-43, $p=0.0289$; for $\mathrm{A} \beta 42$ study, $\mathrm{A} \beta+\mathrm{TDP}$ vs. buffer, $p=0.0019, \mathrm{~A} \beta+\mathrm{TDP}$ vs. $\mathrm{A} \beta, p=0.0311, \mathrm{~A} \beta+\mathrm{TDP}$ vs. TDP-43, $p=0.0311)$. The result indicated that TDP-43-induced $A \beta$ significantly impaired spatial learning and memory of mice to a greater extent than $A \beta$ or TDP43 does.

TDP-43 impairs spatial memory, increases amyloid plaques, interacts with $A \beta$, and induces microgliosis in APP/PS1 $\Delta \mathrm{E} 9$ mice. To further investigate the effect of TDP-43 on AD-related pathology, we bilaterally injected TDP-43 protein into the hippocampus of $\mathrm{APP} / \mathrm{PS} 1 \triangle \mathrm{E} 9$ mice and examined the resulting behavior and pathology. We first performed the MWM for 6month-old APP/PS1 $\triangle \mathrm{E} 9$ mice and their wild-type littermates that received TDP-43 injection at the age of 5 months. In the pretraining phase, all the groups exhibited a similar time-dependent decrease in escape latency, demonstrating that the sample had no injection effect (Supplementary Fig. 7a, repeated two-way ANOVA, time factor: $F=35.26$; df $1 / 27 ; p<0.001$; treatment factor, $F=1.964$; df $3 / 27 ; p=0.143)$. The swim speeds did not differ among the groups (Supplementary Fig. 7b, one-way ANOVA, $F=1.746$, df $3 / 27, p=0.181)$. The results demonstrated that the visual ability and navigated motivation of all the groups were not impaired. In the training phase, the escape latency time of TDP-43-injected APP/PS1 $\triangle \mathrm{E} 9$ group was significantly increased compared with that of the buffer-injected group (Fig. 6a, repeated two-way ANOVA, time factor: $F=8.291$, df $4 / 44, p<0.0001$; TDP-43 factor: $F=24.03$; df $1 / 11 ; p=$ $0.0005)$. No difference was observed in the TDP-43-injected and buffer-injected wild-type mice (repeated two-way ANOVA, time factor: $F=24.72$, df $4 / 60, p<0.0001$; TDP-43 factor: $F=1.606$; df $1 / 15 ; p=0.2243)$. In the probe test after the platform was removed, the mice in the TDP-43-injected APP/PS1 $\Delta \mathrm{E} 9$ or WT group spent significantly less time in the target quadrant than the buffer control group did. Furthermore, the memory function of the TDP-43-injected APP/PS1 $\triangle \mathrm{E} 9$ mice was more significantly impaired than that of the TDP-43-injected wild-type mice (Fig. 6b, one-way ANOVA, Holm-Sidak's multiple comparisons, buffer-WT vs. TDP-43-WT, $p=0.0046$; buffer- APP/PS1 $\Delta \mathrm{E} 9$ vs. TDP-43-APP/PS1 $\Delta \mathrm{E} 9, p<0.0001$; TDP-WT vs. TDP-43-APP/ $\mathrm{PS} 1 \Delta \mathrm{E} 9, p=0.0046$; buffer-WT vs. TDP-43-APP/PS1 $\Delta \mathrm{E} 9, p<$ 0.0001; TDP-WT vs. buffer-APP/PS1 $\Delta \mathrm{E} 9, p=0.0046)$. The time spent of the TDP-43-injected APP/PS1 $\triangle \mathrm{E} 9$ in the opposite quadrant was longer than that of the other groups (one-way ANOVA, Holm-Sidak's multiple comparisons, buffer-WT vs. TDP-43-APP/PS1 $\Delta \mathrm{E} 9, \quad p=0.0036$, TDP-43-WT vs. TDP-43$\mathrm{APP} / \mathrm{PS} 1 \Delta \mathrm{E} 9, p=0.012$, buffer-APP/PS1 $\Delta \mathrm{E} 9$ vs. TDP-43-APP/ $\mathrm{PS} 1 \Delta \mathrm{E} 9, p=0.0008)$. Altogether, the results demonstrated that TDP-43 worsens the spatial memory in APP/PS1 $\Delta \mathrm{E} 9$ mice. In the pathological examination, we first analyzed the amyloid plaque burden in different brain areas. The $A \beta$ level was quantified by immunostaining with $\mathrm{A} \beta$ antibody $4 \mathrm{G} 8 / 6 \mathrm{E} 10$. The results showed that TDP-43 injection significantly increased the amyloid plaque burden in the olfactory bulb, the amygdala, the prefrontal cortex, the motor cortex, and the somatosensory cortex (Supplementary Fig. 8, repeated two-way ANOVA, TDP-43 factor: $F=59.94$; df $1 / 56 ; p<0.0001)$. We further analyzed TDP-43 oligomers and A $\beta$ localization in the hippocampus dentate gyrus. We found that TDP-43 oligomers probed by poly TDP-O antibody were mostly intracellular and colocalized majorly with intraneuronal $\mathrm{A} \beta$ 
a

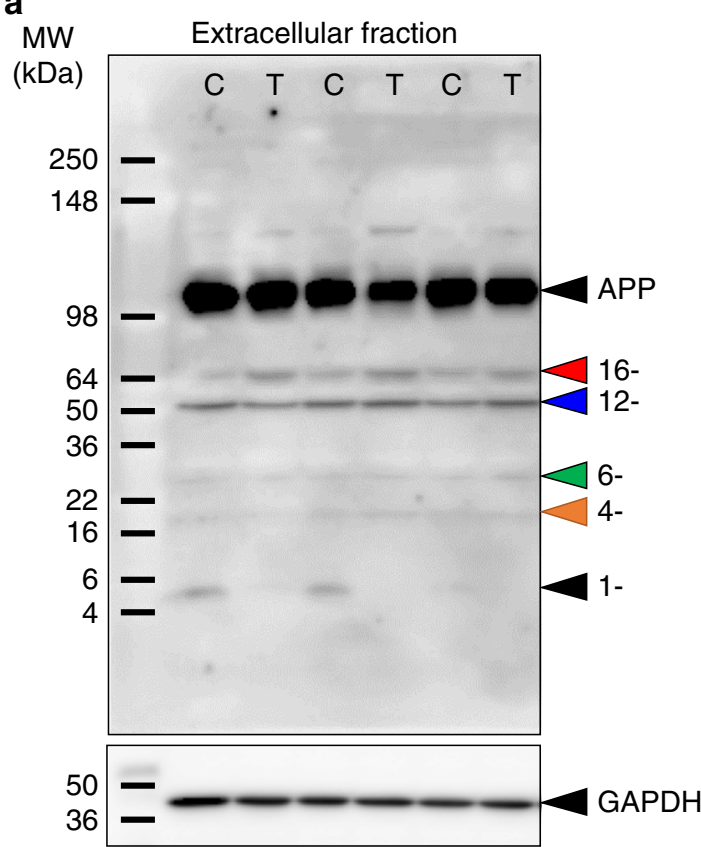

b

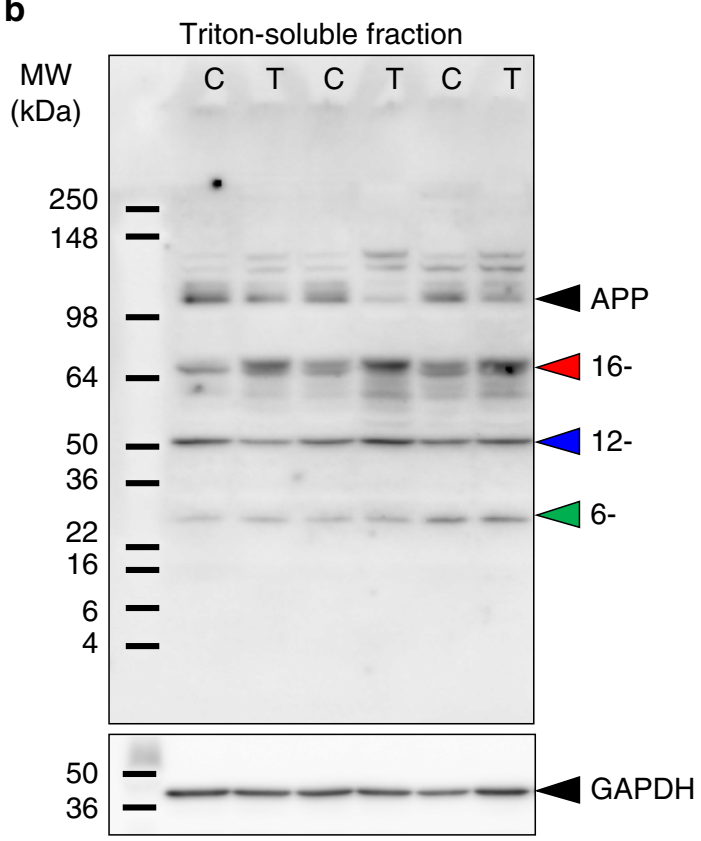

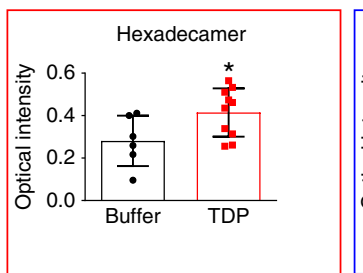
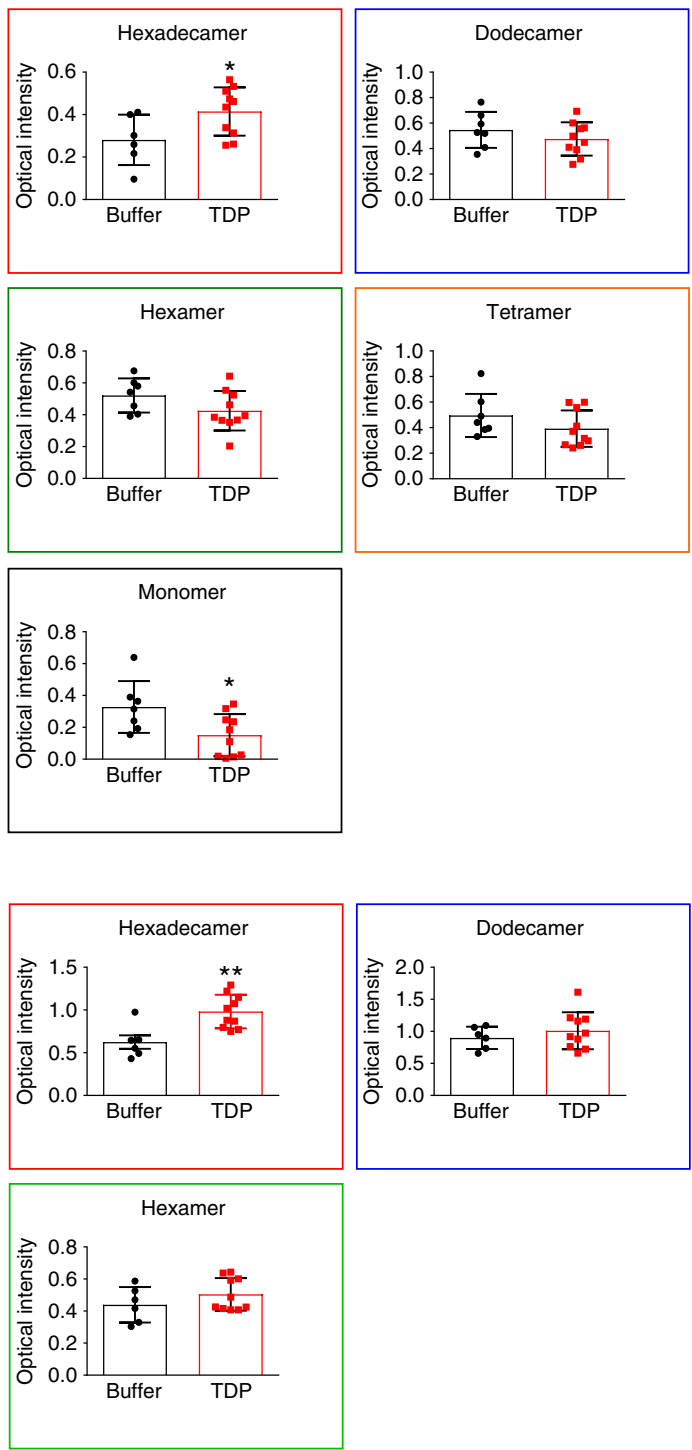

Fig. 7 TDP-43 alters A $\boldsymbol{\beta}$ assembly in APP/PS1 1 E9 mice. Representative western blot of $\mathbf{a}$ extracellular-enriched and $\mathbf{b}$ Triton-soluble fraction of TDP-43injected or buffer-injected APP/PS1 $\triangle$ E9 mice brain. A $\beta$ was detected and quantified in different assembles. TDP-43 group is denoted as T, and the buffer group is denoted as C. The quantitative results of each A $\beta$ assembly in TDP-43-injected $(n=6)$ or buffer-injected $(n=10)$ APP/PS1 $1 \Delta 9$ mice were shown on the right panels. The averaged data and s.e.m. are plotted. The statistical analysis was performed by two-tailed Mann-Whitney test, ${ }^{\star} p<0.05,{ }^{\star \star} p<0.01$ (for extracellular-enriched fraction, Hexadecamer: Buffer vs. TDP-43, $p=0.042$; Monomer: Buffer vs. TDP-43, $p=0.043$; For Triton-soluble fraction, Hexadecamer: Buffer vs. TDP-43, $p=0.005)$. Source data are provided as a Source Data file.

$(\sim 94 \%)$ and a few with amyloid plaques ( 6\%) (Fig. 6c, d). We further conducted immunoprecipitation (IP) to examine the interaction between $A \beta$ and TDP-43 (Fig. 6e) in TDP-43-injected $\mathrm{APP} / \mathrm{PS} 1 \Delta \mathrm{E} 9$ mice. We fractioned the mouse frontal cortex into extracellular-enriched fraction, Triton-soluble fraction, and guanidine hydrochloride ( $\mathrm{GdnHCl}$ )-soluble fraction in accordance with the procedure described in the method. The mixture of $A \beta$ antibodies $6 \mathrm{E} 10$ and $4 \mathrm{G} 8$ was used to pull down $\mathrm{A} \beta$ and the associated proteins in the extracellular-enriched and Tritonsoluble fractions from the frontal cortex. The eluents were probed with a mixture of two rabbit TDP-43 antibodies 10782-2-AP and 12892-1-AP. These antibodies recognize both human and mouse TDP-43. IgG was used as a negative control. The IP data showed that $\sim 43 \mathrm{kDa}$ TDP-43 band, most likely full-length TDP-43, was pulled down with $A \beta$ but not with the negative control in the extracellular-enriched and Triton-soluble fractions. The result demonstrated that TDP-43 interacts with $\mathrm{A} \beta$ in extracellularenriched and Triton-soluble fractions of the APP/PS1 $\triangle \mathrm{E} 9$ mouse frontal cortex. Furthermore, inflammation was examined by Iba1 staining for microgliosis. TDP-43 injection to APP/PS1 $\triangle \mathrm{E} 9$ 
mice resulted in increased microgliosis in the hippocampus dentate gyrus (Fig. 6f, Mann-Whitney test, Buffer-APP/PS1 $\Delta$ E9 vs. TDP-43-APP/PS1 $\triangle \mathrm{E} 9, p=0.008, U=13)$. Overall, these results showed that TDP-43 impairs spatial memory, increases amyloid burden, interacts with $\mathrm{A} \beta$, and induces microgliosis in $\mathrm{APP} / \mathrm{PS} 1 \Delta \mathrm{E} 9$ mice.

TDP-43 alters $A \beta$ assembly in APP/PS1 $\Delta \mathrm{E} 9$ mice. $A \beta$ assembly has been strongly implicated in AD pathogenesis ${ }^{7,33}$. Thus, we investigated whether the injected TDP-43 affects $A \beta$ assembly. We employed western blot analysis to examine the $A \beta$ assembly in different fractions from APP/PS1 $\triangle \mathrm{E} 9$ mouse brains with or without TDP-43 injection. In the extracellular-enriched fraction, we detected $A \beta$ species, including monomer, tetramer, hexamer, dodecamer, and hexadecamer detected by a mixture of 6E10 and $4 \mathrm{G} 8$ antibodies. We found that the level of $\mathrm{A} \beta$ hexadecamer was significantly increased in the TDP-43-injected group (Fig. 7a, Mann-Whitney test, $p=0.042, U=11)$, whereas, the $\mathrm{A} \beta$ monomer decreased (Mann-Whitney test, $p=0.043, U=14$ ). The levels of other $A \beta$ assemblies were not changed (Mann-Whitney test, dodecamer: $p=0.414, U=26$; hexamer: $p=0.088, U=17$; tetramer: $p=0.133, U=19)$. In the Tritonsoluble fraction, we detected $\mathrm{A} \beta$ hexamer, dodecamer, and hexadecamer. Again, the level of $A \beta$ hexadecamer was significantly increased in TDP-43-injected group (Fig. 7b, Mann-Whitney test, $p=0.005, U=5)$. The levels of the other detectable $\mathrm{A} \beta$ assemblies were not changed (Mann-Whitney test, dodecamer: $p=0.492, U=23$; hexamer: $p=0.300, U=20)$. We were unable to perform IP and western blot from the $\mathrm{GdnHCl}$ fractions because the high concentration of $\mathrm{GdnHCl}$ strongly affected the experiments. The result indicated that TDP-43 alters A $\beta$ assembly in $\mathrm{APP} / \mathrm{PS} 1 \Delta \mathrm{E} 9$ mice.

TDP-43 oligomers are present and colocalize with intraneuronal $\mathbf{A} \boldsymbol{\beta}$ in $\mathrm{AD}$ patients. Previously, we found that TDP-43 oligomers are present in FTLD and ALS brains but not in agematched controls. Above in vitro and in vivo studies showed that TDP-43 species, including TDP-43 oligomers interacted and colocalized with $\mathrm{A} \beta$ in the mouse brain. Therefore, to examine whether TDP-43 oligomers exist and colocalize with $\mathrm{A} \beta$ in $\mathrm{AD}$ patients, we applied immunostaining by poly TDP-O antibody or TDP-43 C-terminal antibody and A $\beta$ antibody in the entorhinal cortex of four $\mathrm{AD}$ patients ( 3 male, 1 female) with a mean age of $75.5 \pm 5.6$ years old and a Braak stage of IV or V. In immunostaining analysis by poly TDP-O antibody (Fig. $8 \mathrm{a})$, we found the signal colocalized mostly with intraneuronal $\mathrm{A} \beta(\sim 68 \%)$ and little with amyloid plaques $(\sim 7 \%)$. About $25 \%$ of TDP-43 oligomer signal was not colocalized with $\mathrm{A} \beta$. In immunostaining analysis by TDP-43 C-terminal antibody that recognized both native and misfolded TDP-43 (Fig. $8 \mathrm{~b}$ ), we found that about $38 \%$ of the TDP-43 signal colocalized with A $\beta$ in which most signal colocalized with intraneuronal $\mathrm{A} \beta(\sim 37 \%)$ and little with amyloid plaques $(\sim 1 \%)$. A large portion of the signals resided in the nucleus that is not colocalized with $A \beta$. We further used the neuronal marker MAP-2 to identify neurons via immunofluorescence. The result showed that $\mathrm{A} \beta$ and TDP-43 oligomers colocalized dominantly in the cytoplasm of neuronal cells (Fig. 9). The specificity of $A \beta$ was also demonstrated by $A \beta 40$ and $A \beta 42$ specific antibodies (Supplementary Fig. 9a, b, respectively). We found that about $80 \%$ of the TDP-43 oligomer signal colocalized with $A \beta 40$ and $A \beta 42$ at a similar ratio. The TDP-43 oligomer signal mostly colocalized with intracellular $A \beta(\sim 78 \%)$ and slightly colocalized with amyloid plaques $(\sim 2 \%)$. No significant difference between $A \beta 40$ and $A \beta 42$ was observed. These results
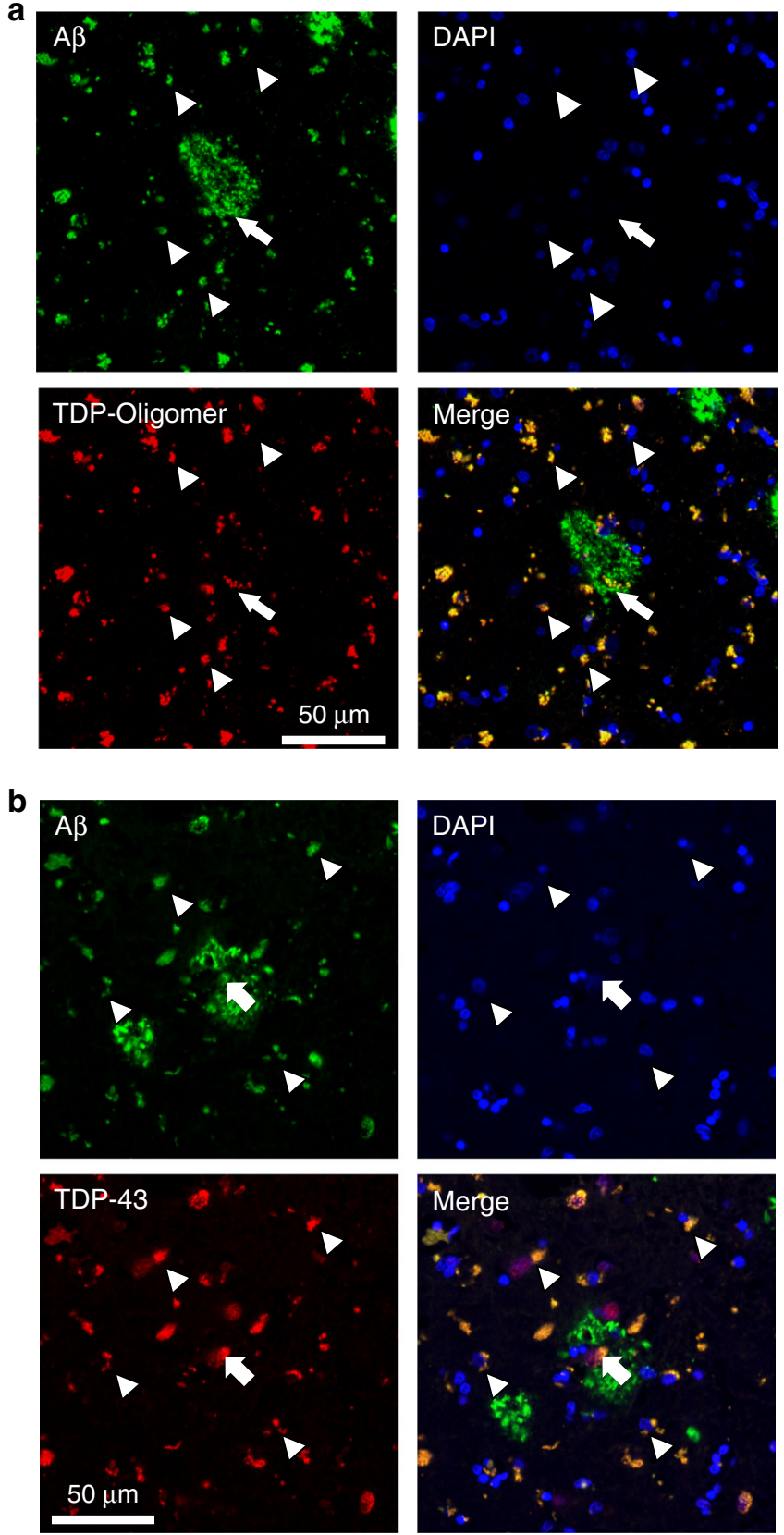

Fig. 8 TDP-43 oligomers colocalize with amyloid plaques in the brain of an AD patient. a Representative immunostaining micrographs reveal that TDP-43 oligomers mainly colocalized with intraneuronal A $\beta$ (arrowhead) and partly with amyloid plaque (arrow). b Representative immunostaining micrographs show that both amyloid plaque (arrow) and intraneuronal $A \beta$ (arrowhead) colocalized with the total TDP-43 in the entorhinal cortex of a 77-year-old patient with Braak stage IV AD. Four induvial samples were performed. Source data are provided as a Source Data file.

clearly showed that TDP-43 oligomers existed and colocalized mostly with intraneuronal $\mathrm{A} \beta$ in the brain of $\mathrm{AD}$ patients.

More $A \beta$ oligomers are present in AD patients with TDP-43 pathology. We further used the hippocampal tissues of several cases with AD to examine their TDP-43 pathology as indicated by hyperphosphorylated TDP-43 (Supplementary Fig. 10b) in western blot and examined the level of $A \beta$ assembly (Supplementary Fig. 10a). We found that the levels high-molecular-weight $A \beta$ 

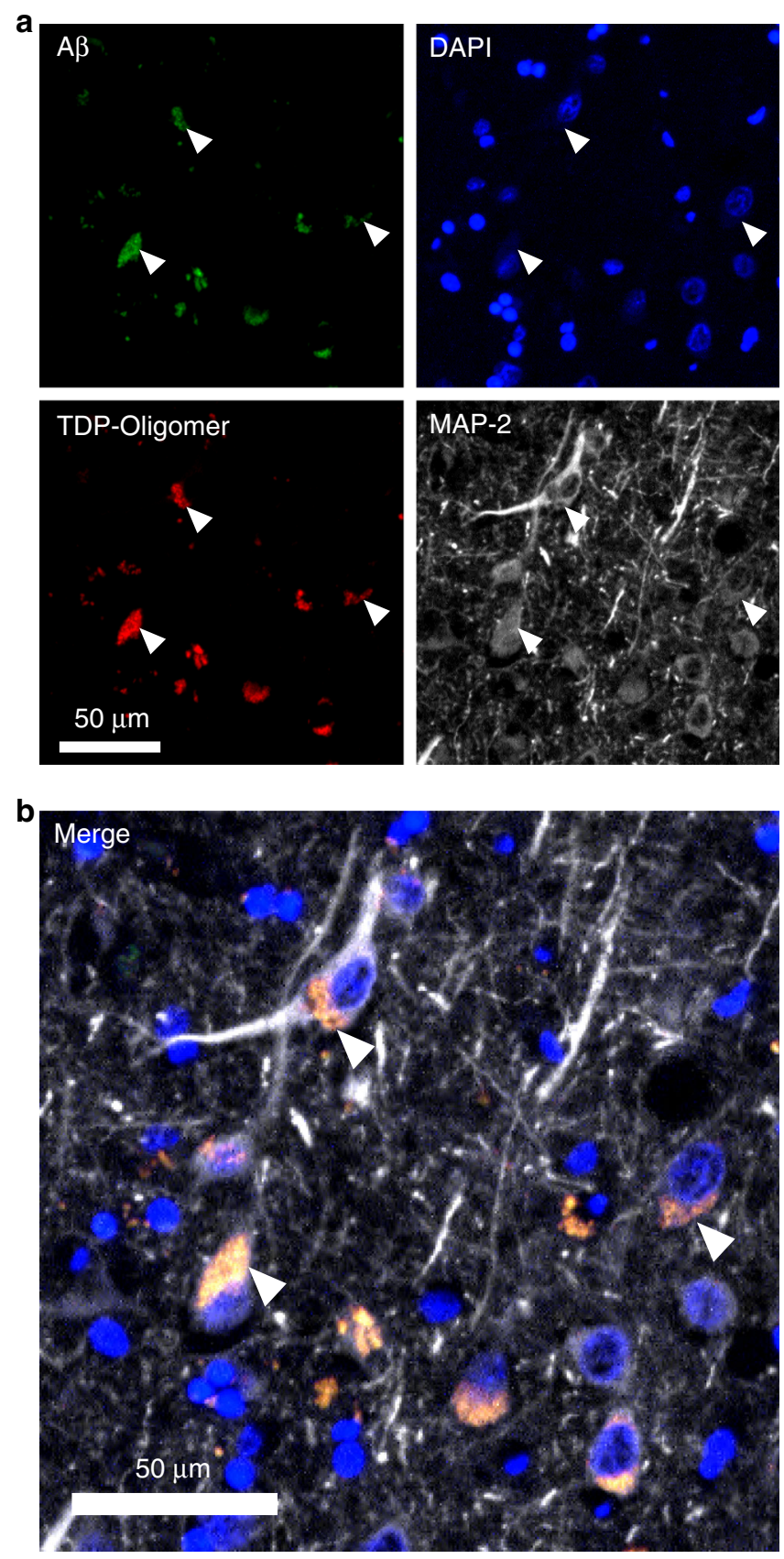

Fig. 9 TDP-43 oligomers colocalize with A $\beta$ in the cytoplasmic region of neurons of an AD patient. Representative immunostaining micrographs reveal that intraneuronal $A \beta$ (arrowhead) colocalize with TDP-43 in the entorhinal cortex of a 77-year-old patient with Braak stage IV AD. a Specimens are stained with $A \beta$ antibodies (4G8 and 6E10), TDP-43 oligomer antibody, neuronal marker MAP-2, and DAPI. b The merged image is shown. The results demonstrated that many TDP-43 oligomers and $A \beta$ colocalized in the cytoplasm of neuronal cells. Four induvial samples were performed. Source data are provided as a Source Data file.

species of $\mathrm{AD}$ patients with hyperphosphorylated TDP-43 $(n=3)$ were lower and their levels of $\mathrm{A} \beta$ oligomers were higher than those of $\mathrm{AD}$ patients but without TDP-43 pathology $(n=5) . \mathrm{A} \beta$ oligomer at $50 \mathrm{kDa}$ was significantly higher in $\mathrm{AD}$ patients with TDP-43 pathology than in AD patients but without TDP-43 pathology. These results were consistent with those observed in $\mathrm{APP} / \mathrm{PS} 1 \Delta \mathrm{E} 9$ mice injected with TDP-43.

\section{Discussion}

$A \beta$ progressively aggregates into amyloid fibril via a nucleationelongation mechanism. In this study, we found that TDP-43 oligomers affected the conformational change in $A \beta$ and prevented the formation of $A \beta$ fibrils. This condition may be attributed to the amyloidogenic properties of TDP-43 since a similar phenomenon has also been observed in other amyloidogenic protein-protein interaction ${ }^{34,35}$. For example, cystatin $\mathrm{C}$ (CysC) forms soluble amyloid oligomers that inhibit $\mathrm{A} \beta$ aggregation more potently than monomeric and dimeric CysC $\mathrm{do}^{34}$. Transthyretin tetramer suppresses $\mathrm{A} \beta$ aggregation and ameliorates $\mathrm{AD}$ phenotype by interacting with $\mathrm{A} \beta^{35}$. TDP-43 possibly formed a complex with $\mathrm{A} \beta$ and caused detrimental effects although only $1 \%$ TDP- 43 was present in the A $\beta$ samples under our experimental conditions. However, we encountered a technical difficulty in separating a defined aggregated species and maintaining the original property because TDP-43 and A $\beta$ both form oligomers. We further found that the inhibition of $A \beta$ fibrillization by TDP-43 only occurs in the early and intermediate stages of $A \beta$ fibrillization, but not in the fibrillar stage and the seeding reaction. The result demonstrated that TDP-43 does not affect mature $A \beta$ fibrils and cannot block fibril growth once the fibril nucleus was added. It also suggests that TDP-43 cannot block secondary nucleation in $\mathrm{A} \beta$ fibrillization. For the discrepancy found in the inhibitory effect of TDP-43 on A $\beta 40$ and $\mathrm{A} \beta 42$, A $\beta 42$ may not be effectively inhibited by TDP-43 because A $\beta 42$ initially forms several oligomers with few monomers and rapidly fibrillizes ${ }^{36-38}$. Furthermore, $A \beta 40$ and $A \beta 42$ oligomerize through distinct pathways ${ }^{36-38}$. Freshly prepared $A \beta 40$ predominantly forms monomers ${ }^{38}$, forms a monomer-added mixture after crosslinking ${ }^{36}$, and slowly fibrillizes ${ }^{6,38,39}$. By contrast, freshly prepared $\mathrm{A} \beta 42$ forms paranuclei ${ }^{36,37}$ or tetramers ${ }^{37,38}$ in equilibrium with monomer, and rapidly aggregates. Therefore, the potential interaction site and species of $A \beta 40$ and $A \beta 42$ for TDP-43 can vary.

In the characterization of the specificity and interaction of TDP-43 and A $\beta$, we found full-length TDP-43, TDP-43_265, and TDP-43_N-term, not TDP-43_RRM $1+2$, potently inhibited A $\beta$ fibrillization; however, TDP-43_RRM1 +2 has strong interaction with A $\beta$. Since full-length TDP-43 formed oligomers and TDP$43 \_265$ and N-term formed dimeric species, the result might indicate that oligomerization/dimerization is required for the inhibitory activity, where the interaction may be majorly attributed from RRM1 +2 and minorly, if any, from $\mathrm{N}$-terminal region. Therefore, we hypothesized that TDP-43 mainly interacted with $A \beta$ through RRM domains and required dimeric/oligomeric TDP-43 to inhibit $A \beta$ fibrillization. This inhibition resulted in the retention of A $\beta$ oligomers. TDP-43_265 was likely present because of the calpain cleavage of TDP-43 in the brain of $\mathrm{AD}$ patients, considering that calpain is implicated in $\mathrm{AD}^{40}$ and calpain-cleaved TDP-43 was found in the brain and spinal cord of ALS patients ${ }^{41}$.

In the animal studies, we demonstrated that the modification of $\mathrm{A} \beta$ aggregation by TDP-43 increased neurotoxicity by impairment of synapse transmission in LTP reduction ex vivo and spatial memory in wild-type mice in vivo. In TDP-43injected APP/PS1 $\triangle \mathrm{E} 9$ mice, TDP-43 impaired spatial memory and increases $A \beta$-related pathologies, including amyloid plaque burden, $A \beta$ assembly, and microgliosis. The result was consistent with previous studies showing that TDP-43 overexpression induces neuroinflammation in a lentiviral gene transfer rat model $^{24}$ and that depletion of TDP-43 in APP/PS1 1 E9 mice decreases the amyloid plaque burden in hippocampus and cortex ${ }^{42}$. Although we found TDP-43 inhibits $A \beta$ fibrilization in vitro, we suggest that brain inflammation induced by TDP-43 injection leads to an increase of the amyloid plaques in vivo. Our 
studies showed that TDP-43 plays multiple roles in $\mathrm{AD}$; for example, TDP- 43 induces more A $\beta$ oligomers by directly interacting with $A \beta$, leading to synaptic dysfunction and memory impairment; TDP-43 increases brain inflammation that worsens spatial memory and causes an increase in amyloid burden ${ }^{43,44}$. TDP-43 also affects APP metabolism ${ }^{45}$. These events could occur in the same periods, but they affected $\mathrm{AD}$ pathology to different degrees.

We showed that TDP-43 oligomers colocalized largely with intraneuronal $\mathrm{A} \beta$ and some with amyloid plaques in APP/ PS1 $\triangle \mathrm{E} 9$ mice and AD brain. TDP-43 interacted with $A \beta$ in both the extracellular-enriched and Triton-solution fractions of APP/ PS1 $\triangle \mathrm{E} 9$ mouse brain after TDP-43 injection. We suggest that soluble $A \beta$ oligomers in the extracellular-enriched and Tritonsolution fractions interacted with TDP-43 since amyloid plaques were retained in $\mathrm{GdnHCl}$ fractions. Intracellular $\mathrm{A} \beta$ has been observed in both sporadic and familial AD patients ${ }^{46,47}$ and was reported to be an early event in $\mathrm{AD}$ and in Down syndrome preceding extracellular $A \beta^{47,48}$. The interaction site for TDP-43 and $A \beta$ in cell could be in cytoplasm and/or lysosome. $A \beta$ and TDP-43 have been reported to influence endosomal-lysosomal pathways ${ }^{49,50}$ where both aggregates can be cleared by autophagy mechanism ${ }^{51,52}$. Extracellular $A \beta$ can be re-internalized and found inside endosomal/lysosomal compartments ${ }^{53}$. Cytosolic $A \beta$ can also be found without vesicle structure ${ }^{54}$. Passive leakage along the pathways and active uptake of extracellular $A \beta$ by several cell surface receptors, leading to intracellular accumulation have been reported ${ }^{9}$. Intracellular $A \beta$ has been reported to bind to several cytosolic chaperons like $\mathrm{Hsp} 70$ and small heat shock proteins ${ }^{55}$, cytosolic enzymes such as SOD1 ${ }^{56}$, and directly interact with amyloid binding alcohol dehydrogenase in mitochondria ${ }^{57}$.

Furthermore, in our TDP-43-injected APP/PS1 $\triangle \mathrm{E} 9$ mice, a specific $A \beta$ assembly hexadecamer is significantly increased. Previous studies showed that injection of $A \beta$ dodecamers impairs the memory of young rats ${ }^{33}$, and $\mathrm{A} \beta$ dimers isolated from $\mathrm{AD}$ brains impair synaptic plasticity and memory ${ }^{58}$. These studies have highlighted the importance of specific oligomeric $A \beta$ for memory impairment. Thus, we propose that the spatial memory impairment in TDP-43-injected APP/PS1 $\triangle \mathrm{E} 9$ mice is due to increased inflammation and accumulation of $A \beta$ oligomers, which is considered more toxic than $A \beta$ fibrils and monomers ${ }^{7}$. However, although we showed that TDP-43 induced both A $\beta 40$ and $A \beta 42$ toxicity in LTP and in water maze study in the injection mice model and demonstrated that both $\mathrm{A} \beta 40$ and $\mathrm{A} \beta 42$ colocalized in brain of post mortem $\mathrm{AD}$ patients, the exact molecular interaction of TDP-43 to $A \beta 40$ and $A \beta 42$ needs to be further investigated to elucidate potential differences resided in $A \beta 40$ and $A \beta 42$. Overall, our results demonstrated that TDP-43 inhibited $A \beta$ fibrillization by interacting with $A \beta$ and exacerbated $A D$ related pathology.

\footnotetext{
Methods

TDP-43 cloning, expression, and purification. For TDP-43 proteins, the plasmids used for expression are TDP-43_FL, a pET14b vector containing cDNA of full-length TDP-43, TDP-43_265 (a pET14b vector containing cDNA of TDP-43 aa. 1-265), TDP-43_N-term (a pQE30 vector containing the cDNA of TDP-43 aa. 1-101), and TDP-43_RRM1 + 2 (a pQE30 vector containing the cDNA of TDP-43 aa. 101-265). Full-length TDP-43 was cloned previously ${ }^{22}$. TDP- 43 -265 was cloned by deletion mutagenesis of full-length TDP-43 with forward primer and reverse primer listed in Supplementary Table 1. TDP-43_N-term and TDP-43_ RRM1 +2 are gifts from Dr. Hanna S. Yuan, Institute of Molecular Biology, Academia Sinica ${ }^{59}$. The pET14b plasmids were transformed into E. coli strain Rosetta 2 (Novagen, Merck KGaA), and the pQE30 plasmids were transformed into E. coli strain M15. All TDP- 43 proteins contain His-tag in the N-terminal region. Full-length TDP-43 and TDP- 43_265 contain extra N-terminal residues MGSSHHHHHHSSGLVPRGSHMLE. TDP-43_N-term and TDP-43_RRM1 + RRM2 contained extra N-terminal residues MRGSHHHHHHGS. E. coli were
}

grown in LB medium with $50 \mu \mathrm{g} / \mathrm{ml}$ ampicillin at $37^{\circ} \mathrm{C}$ to OD600 around 0.4 and induced by $0.5 \mathrm{mM} \mathrm{IPTG}$ at $16^{\circ} \mathrm{C}$. After $22 \mathrm{~h}$, the cells were harvested and lysed in $30 \mathrm{mM}$ Tris- $\mathrm{HCl}$ buffer, $\mathrm{pH}$ 8, containing $500 \mathrm{mM} \mathrm{NaCl}, 10 \%$ glycerol, $1 \mathrm{mM}$ dithiothreitol (DTT), $2 \%$ RNase A, $\%$ DNase I, and protease inhibitor cocktail (Complete, EDTA-free, Roche Applied Science, Mannheim, Germany). The supernatant was collected after centrifugation and loaded onto a Ni- NTA affinity column (GE Healthcare Bio-Sciences AB, Uppsala, Sweden). The column was first equilibrated using a buffer containing $30 \mathrm{mM}$ Tris, $\mathrm{pH} 8,500 \mathrm{mM} \mathrm{NaCl}, 1 \mathrm{mM}$ DTT, $20 \mathrm{mM}$ imidazole, and $10 \%$ glycerol. Proteins were eluted with a step gradient of imidazole in the same running buffer. The purity of His-tagged TDP-43 protein was checked on SDS-PAGE and identified by Coomassie blue staining. Proteins were stored at $-20^{\circ} \mathrm{C}$ freezer and further dialyzed with $10 \mathrm{mM}$ Tris at $\mathrm{pH}$ 8 right before the experiments. The dialyzed protein was centrifuged at $17,000 \times g$ at $4{ }^{\circ} \mathrm{C}$ for $30 \mathrm{~min}$ to remove all precipitates. Protein concentration was quantified by micro BCA protein assay kit (Thermo Fisher Scientific, USA).

A $\boldsymbol{\beta}$ preparation. Recombinant $A \beta$ expression and purification were performed following our previous study ${ }^{60}$. No additional amino acid was generated. The purified $A \beta$ peptide was lyophilized and stored at $-80^{\circ} \mathrm{C}$. Biotinylated $\mathrm{A} \beta$ was purchased from Biopeptide Inc. (San Diego, CA, USA).

ThT assay. For ThT assay shown in Fig. 1a, $\mathrm{A} \beta 40$ powder, $0.1 \mathrm{mg}$, was first treated with hexafluoroisopropanol (HFIP), lyophilized, then dissolved in $100 \mu \mathrm{L}, 3 \mathrm{mM}$ $\mathrm{NaOH}$ and lyophilized. The lyophilized powder was then dissolved in $100 \mu \mathrm{L}$, $10 \mathrm{mM}$ Tris buffer, $\mathrm{pH} 7.8$, and $\mathrm{A} \beta$ stock concentration was determined via BCA assay. The $\mathrm{A} \beta$ alone sample was prepared from a stock solution by adding more Tris buffer to reach $25 \mu \mathrm{M}$. As the concentration of freshly dialyzed TDP-43_FL solution is very low $(\sim 0.25 \mu \mathrm{M}$ quantified by Micro BCA assay), TDP-43_FL in $10 \mathrm{mM}$ Tris buffer, $\mathrm{pH} 7.8$ was directly added into another $0.1 \mathrm{mg} \mathrm{A} \beta$ powder to avoid the decrease of concentration of TDP-43. This condition did not contain any organic solvent or denaturant. The samples were placed in Eppendorf tubes and shaken for $20 \mathrm{~s}$ at $500 \mathrm{rpm}$ every h in Thermomixer C (Eppendorf, Germany) at $25^{\circ} \mathrm{C}$. The selected time point samples were collected for ThT measurement at $25^{\circ} \mathrm{C}$.

For ThT assay shown in Fig. 1e-h and Fig. 2, A $\beta 40$ peptide at $0.1 \mathrm{mg}$ was treated with HFIP and lyophilized for at least $18 \mathrm{~h}$ before use. A $\beta 40$ sample was dissolved in $6 \mu \mathrm{l}$ dimethyl sulfoxide (DMSO) and diluted into $94 \mu \mathrm{l}, 10 \mathrm{mM}$ Tris buffer at $\mathrm{pH}$. The concentration of $\mathrm{A} \beta$ stock was determined by BCA protein assay kit (Thermo Fisher Scientific). An aliquot of freshly prepared $A \beta$ stock was diluted into TDP-43 samples or buffer containing $5 \mu \mathrm{M}$ ThT to prepare A $\beta$ solution at $25 \mu \mathrm{M}$. The final DMSO concentration in the samples is less than $2 \%$. To determine the effect of TDP-43 protein on $A \beta$ oligomers or $A \beta$ fibrils, the $A \beta$ stock was first incubated for $20 \mathrm{~h}$ at $4^{\circ} \mathrm{C}$ with continuous shaking at $300 \mathrm{rpm}$ to prepare $A \beta$ oligomers or with continuous shaking at $1000 \mathrm{rpm}$ for $24 \mathrm{~h}$ to prepare $A \beta$ fibrils. The A $\beta$ species were validated in TEM. TDP- 43 and control buffer were added to $A \beta$ oligomer or fibrils and subjected to ThT assay. For seeding experiment, the $2.5 \mu \mathrm{M} \mathrm{A} \beta$ fibrils solution was added at the beginning of aggregation assay of $\mathrm{A} \beta$ at $25 \mu \mathrm{M}$. The samples were incubated in a 384-well opaque microtiter plate, sealed with a transparent film, and monitored by a microplate reader (SpectraMax M5, Molecule Devices) at $25^{\circ} \mathrm{C}$. The ThT signals were measured every hour with a 60 -s mixing before the measurement. ThT fluorescence was excited at $442 \mathrm{~nm}$, and the emission spectrum was collected at $485 \mathrm{~nm}$ by software SoftMax Pro 6.3 in SpectraMax M5. Data were plotted and analyzed in GraphPad Prism 7.0.

Dot blotting. To probe the conformational change of $\mathrm{A} \beta 40$ during fibrillization, $2 \mu \mathrm{L}$ of $\mathrm{A} \beta$ samples with and without TDP-43 were collected from different time points from the ThT study shown Fig. 1a. They were dotted onto nitrocellulose membranes and probed by anti-amyloid oligomers A11 polyclonal antibody (1:1000, Life Technologies) and anti-amyloid fibrils OC polyclonal antibody (1:10,000, Merck Millipore). For full-length TDP-43, freshly dialyzed TDP-43_FL and variants were prepared at the aforementioned condition and the concentration was determined via micro BCA assay. Two microliters of TDP- 43 variants at $0.25 \mu \mathrm{M}$ were dotted onto nitrocellulose membranes. After blocking with $5 \%$ skim milk in TBST, the membrane was blotted with polyclonal TDP-43 oligomer-specific antibody, poly TDP-Oำ (1:4000 in blocking solution) and anti-rabbit horseradish peroxidase (HRP)-conjugated secondary antibodies (1:5000, Merck Millipore).

Circular dichroism. The time-course samples collected for $\mathrm{CD}$ measurement were prepared in the same condition as for the ThT assay in Fig. 1a. All far-ultraviolet CD spectra were recorded by Spectra Manager 2.0 in Jasco J-815 spectropolarimeter (Jasco Inc.) using a circular quartz cell (Hellma) with $1 \mathrm{~mm}$ path length at room temperature (RT). Each spectrum was collected from 250 to $190 \mathrm{~nm}$, corrected with buffer background, and averaged from 10 scans. Data were plotted in GraphPad Prism 7.0.

Transmission electron microscopy. Five microliters of TDP- 43 proteins or A $\beta$ samples from ThT assay were deposited onto 400-mesh Formvar carbon-coated copper grids (EMS electron Microscopy Sciences, Hatfield, PA, USA) for 1 min. 
The grids were blotted, washed with droplets of Milli-Q water, and stained with $2 \%$ uranyl acetate. The samples were examined with a FEI Tecnai G2 F20 Super TWIN transmission electron microscope with an accelerating voltage of $120 \mathrm{kV}$. For immunogold labeling, $5 \mu \mathrm{l} \mathrm{A} \beta$ fibrils with TDP- 43 was placed on grids for $5 \mathrm{~min}$, then washed, blocked, and probed following previous protocol ${ }^{22}$ but with primary antibody using anti-TDP-43 N-term rabbit antibody (10782, 1:1000, Proteintech). In the end, the grids were incubated with an $18 \mathrm{~nm}$ gold-conjugated secondary anti-rabbit IgG antibody (1:40, Jackson ImmunoResearch) at RT for $1 \mathrm{~h}$. The unbound antibody was heavily washed by high-salt Tween and PBS. The grids were then fixed by $1 \%$ glutaraldehyde in PBS at RT for 10 min and washed six times by double-distilled $\mathrm{H}_{2} \mathrm{O}$. Finally, the grids were negatively stained using $2 \%$ uranyl acetate.

Enzyme-linked immunosorbent assay. The concentration of freshly purified TDP-43_FL and truncation proteins were quantified by Bradford protein assay (Thermo Fisher Scientific) and the same mole number of each variant at $1 \mu \mathrm{M}(200$ pmole in $200 \mu \mathrm{L}$ ) was immobilized in 96-well ELISA microplates (Thermo Fisher Scientific, USA) by incubation overnight at $4{ }^{\circ} \mathrm{C}$. HFIP and $\mathrm{NaOH}$-treated biotinlabeled $\mathrm{A} \beta 40$ was prepared in $10 \mathrm{mM}$ Tris at $\mathrm{pH} 7.8$ to make a series of $\mathrm{A} \beta$ solution with concentrations ranging from 0 to $38 \mu \mathrm{M}$. After immobilization, the plate was blocked for $2 \mathrm{~h}$ at RT with $5 \%$ skim milk in TBST ( $50 \mathrm{mM}$ Tris-Cl, pH 7.6, $150 \mathrm{mM}$ $\mathrm{NaCl}, 0.1 \%$ Tween-20). The plates were washed and probed by biotin- $\mathrm{A} \beta 40$ solution for $2 \mathrm{~h}$ at RT. After wash, the plates were subjected to HRP-conjugated Streptavidin (1:5,000, Merck Millipore) for $1 \mathrm{~h}$ at RT. After another wash, the color was developed by adding $100 \mu \mathrm{L}$ 3,3, 5,5 -tetramethyl benzidine (Merck Millipore). The reaction was stopped with $100 \mu \mathrm{L} 250 \mathrm{mM} \mathrm{HCl}$ and the absorbance was recorded at $450 \mathrm{~nm}$ by software SoftMax Pro 6.3 in SpectraMax M5 (Molecular Device). Data were plotted and analyzed in GraphPad Prism 7.0.

Biolayer interferometry analysis. HFIP- and $\mathrm{NaOH}$-treated N-terminally biotinylated A $\beta 40$ was dissolved in $10 \mathrm{mM}$ Tris, $\mathrm{pH} 7.8$ with $0.005 \%$ Tween- 20 to make a concentration of $10 \mu \mathrm{M}$ solution. The sensors used are streptavidin-coated (SA sensor, ForteBio). The sample sensors and reference sensors were also preincubated in the same buffer before loading. The biotinylated $A \beta 40$ was immobilized to sensors and then the sensors were subjected to TDP-43_FL or TDP-43 variants solution to probe the interaction between $\mathrm{A} \beta$ and TDP- 43 proteins. In general, all steps were performed at $37^{\circ} \mathrm{C}$ with continuous agitation at $1,000 \mathrm{rpm}$. The association and dissociation time were both $300 \mathrm{~s}$. The regeneration buffer was $10 \mathrm{mM}$ glycine at $\mathrm{pH}$ 1.5. The sensorgrams were measured with double references (reference buffer and reference sensor) using an Octet Red 96 and $K_{\mathrm{D}}$ value was obtained by fitting the data using the Data Analysis Software (ForteBio).

SDS-PAGE and western blot for proteins. The ThT assays were stopped by adding SDS-PAGE sample buffer and then the samples without heating were loaded onto a $13.3 \%$ Tris/tricine separating gel with $10 \%$ and $4 \%$ stacking portions. Separated $A \beta$ species on the gel were electrophoretically transferred to a nitrocellulose membrane at $250 \mathrm{~mA}$ for $75 \mathrm{~min}$ at $4^{\circ} \mathrm{C}$. The membrane was blocked with $5 \%$ skim milk in TBST. A $\beta$ samples were probed by $6 \mathrm{E} 10$ antibody (1:5000, BioLegend) and anti-mouse IgG secondary antibody. The signals were visualized by ECL detection kit (Merck Millipore).

Animal. All experiments were done in accordance with the National Institutes of Health Guideline for Animal Research (Guide for the Care and Use of Laboratory Animals) and Taiwan Animal Protection Law and were approved by the Academia Sinica Institutional Animal Care and Utilization Committee (IUCAC 16-02-939). C57BL/6 mice were obtained from the National Laboratory Animal Center (Taipei, Taiwan) and the APP/PS1 $\triangle \mathrm{E} 9$ (B6C3-Tg(APPswe,PSEN1dE9)85Dbo/Mmjax) mice were obtained from the Jackson Laboratory (Bar Harbor, ME, USA). All of the APP/PS1 $\triangle \mathrm{E} 9$ mice were genotyped using a protocol provided by the Jackson Laboratory. All of the mice were housed (4-5 per cage) at a stable temperature $\left(23 \pm 1{ }^{\circ} \mathrm{C}\right)$, humidity $55 \pm 5 \%$, and unrestricted access to food and water with a light cycle from $7 \mathrm{am}$ to $7 \mathrm{pm}$ during the experimental period. We used 3-4 mice per group for the electrophysiology study, 5-9 C57/BL6JNarl mice per group and 6-8 APP/PS1 $\triangle \mathrm{E} 9$ mice per group for the Morris Water Maze (MWM), $3 \mathrm{APP} /$ $\mathrm{PS} 1 \Delta \mathrm{E} 9$ mice per group for the immunostaining staining, 9-10 APP/PS1 $\triangle \mathrm{E} 9$ mice per group for microgliosis staining, and 6-10 APP/PS1 $\Delta \mathrm{E} 9$ mice per group were used for $A \beta$ western blotting.

Electrophysiological analysis. Hippocampal slice for field excitatory postsynaptic potential (fEPSP) was prepared as previously described ${ }^{61}$. The 12 -week-old C57BL/ 6JNarl male mice were anaesthetized by isoflurane and decapitated, then the mice brain was quickly removed and placed into the ice-cold artificial cerebrospinal fluid $\left(119 \mathrm{mM} \mathrm{NaCl}, 2.5 \mathrm{mM} \mathrm{KCl}, 1.3 \mathrm{mM} \mathrm{MgSO}_{4}, 2.5 \mathrm{mM} \mathrm{CaCl} 2,26.2 \mathrm{mM} \mathrm{NaHCO}_{3}\right.$, $11 \mathrm{mM}$ Glucose and $1.25 \mathrm{mM} \mathrm{NaH} \mathrm{PO}_{4}$, aCSF). The hippocampus was removed and then cut into $450 \mu \mathrm{m}$ thickness by vibratome (Leica, Nussloch, Germany). The hippocampal slices were recovered for $2 \mathrm{~h}$ at room temperature in a chamber with aCSF, which bubbled with a mixture of $95 \% \mathrm{O} 2$ and $5 \% \mathrm{CO}_{2}$. For extracellular recordings, the hippocampal slices were placed at the center of a MED-P515A/5 $(1 \mathrm{~mm})$ probe (Alpha MED Scientific Inc., Osaka, Japan) with 64 embedded recording electrodes containing with circulating aCSF and $100 \mu \mathrm{M}$ picrotoxin bubbled with a mixture of $95 \% \mathrm{O}_{2}$ and $5 \% \mathrm{CO}_{2}$. The fEPSP was recorded from the Schaffer collaterals fibers on the stratum radiatum layer of hippocampal CA1 area by using MED64 multichannel recording system in Neuro- electrophysiology core, Academia Sinica. Test stimuli were given every $30 \mathrm{~s}(0.033 \mathrm{~Hz})$, and the stimulus intensity was set to produce $40 \%$ of the maximum spike-free response. A stable baseline was recorded for at least $30 \mathrm{~min}$ before treatment with aCSF containing $\mathrm{A} \beta$, full-length TDP- 43 , or TDP-43-induced $\mathrm{A} \beta$ at indicated concentrations. The samples were prepared following $\mathrm{A} \beta$ preparation for ThT assay for Fig. $2^{32}$ and collected at $\sim 50 \mathrm{~h}$ of incubation. After treatment with protein or respective buffer control for $30 \mathrm{~min}$, LTP was induced by theta burst stimulation (10 bursts of 4 stimuli at $100 \mathrm{~Hz}$, with an inter-burst interval of $200 \mathrm{~ms}$ ) given at baseline intensity according to our previous publication ${ }^{61}$. The fEPSP slope was measured from $\sim 10-90 \%$ of the rising phase using a least squares regression. The data were quantified by normalization to the baseline slope and mean \pm SEM are shown. Repeated two-way ANOVA was used for statistical analysis. The respective buffer control was performed on the same mice but different brain slice to exclude individual bias.

Intracranial injection to mice. Five-month-old male C57BL/6JNarl mice or APP/ PS1 $\triangle \mathrm{E} 9$ male mice were anaesthetized by intraperitoneal injection of a mixture of tranquilizer (Zoleti 50, $1 \mathrm{mg} / 10 \mathrm{mg}$ body weight, Vibrac, Amherst, MA, USA), analgesics (Rompun, $10 \mu \mathrm{L} / 10 \mathrm{mg}$ body weight, Bayer, Toronto, Canada). The C57BL/6JNarl mice received bilateral intrahippocampal injection of $2 \mu \mathrm{L}$ recombinant $\mathrm{A} \beta 40$ or $\mathrm{A} \beta 42(25 \mu \mathrm{M})$, full-length TDP-43 $(0.25 \mu \mathrm{M})$, or TDP-43-induced $\mathrm{A} \beta(25 \mu \mathrm{M}$ A $\beta 40$ or $\mathrm{A} \beta 42$ with $0.25 \mu \mathrm{M}$ TDP- 43). APP/PS1 $\Delta \mathrm{E} 9$ mice received bilateral intrahippocampal injection of $2 \mu \mathrm{L}$ recombinant full-length TDP-43 $(2.5 \mu \mathrm{M})$. The stereotaxic coordinates of the injection site are in relation to bregma as follows: Posterior $2 \mathrm{~mm}$; mediolateral $1 \mathrm{~mm}$; ventral $2 \mathrm{~mm}$. The injection needle was slowly approached to the desired depth and the mentioned protein was injected using a microsyringe $(0.1 \mu \mathrm{L} / \mathrm{min}, 32$-gauge Hamilton Company, NV, USA). The needle left in place for an additional $5 \mathrm{~min}$ to limit the diffusion of the injected protein.

Morris water maze test. The MWM was performed in a custom-made circular pool with a diameter of $154 \mathrm{~cm}$ and a wall height of $60 \mathrm{~cm}$, which was filled with opaque water (diluted milk) at a temperature of $20 \pm 2^{\circ} \mathrm{C}$ and depth of $32 \mathrm{~cm}$. During the pre-training trials, the circular escape platform made of transparent Plexiglas (diameter $13 \mathrm{~cm}$ ) was emerged $0.3 \mathrm{~cm}$ above the water surface and kept location constant for 2 days. In the training trial, the escape platform was submerged $0.5 \mathrm{~cm}$ below the water surface and the location of the hidden platform was moved to another place and kept constant for five days. Animals were given a session training $(1,000-1,800 \mathrm{~h})$ per day during pre- and training trails. Each session consisted of four swim trials (120 s per trial) with different quadrant starting positions for each trial. After the last day of training, the mice were given a probe test. During the probe test, animals were placed in the center position of the pool, and the mice were allowed to swim for $60 \mathrm{~s}$ without the platform presented. The whole process was recorded by a charge-coupled device camera and the escape latency (i.e., time to reach the platform, in seconds), time spending in target quadrant of probe test, path length and swim speed $(\mathrm{cm} / \mathrm{s})$ were analyzed by EthoVision video tracking system (Noldus Information Technology, Wageningen, Netherlands).

Brain tissue preparation and fractionation. The mice were anesthetized by intraperitoneal injection of a mixture of tranquilizer (Zoleti $50,1 \mathrm{mg} / 10 \mathrm{mg}$ body weight, Vibrac, Amherst, MA, USA), analgesics (Rompun, $10 \mu \mathrm{L} / 10 \mathrm{mg}$ body weight, Bayer, Toronto, Canada) and perfused from the left ventricle with ice-cold PBS, and their brains were quickly removed. The left hemispheres were stored in $-80{ }^{\circ} \mathrm{C}$ immediately. The right hemispheres were fixed in $10 \%$ formalin at $4{ }^{\circ} \mathrm{C}$ for frozen section and subjected to immunostaining. The brain was cut into an average of 130 pieces of $30-\mu \mathrm{m}$ coronal sections. For analysis of amyloid plaque burden or TDP-43 oligomer signals, the brain sections were measured in every 12th section of the brain from anterior (stereotaxic reference: bregma $2.5 \pm 0.2 \mathrm{~mm}$ ) to posterior part (stereotaxic reference: bregma $-6.5 \pm 0.2 \mathrm{~mm}$ )

The left hemispheres were collected, homogenized, and subjected to different fractionations following previous literature ${ }^{33}$. For extracellular-enriched protein fraction, $25 \%(\mathrm{w} / \mathrm{v})$ lysis buffer containing $50 \mathrm{mM}$ Tris- $\mathrm{HCl}(\mathrm{pH}$ 7.6), $0.01 \% \mathrm{NP}-$ $40,150 \mathrm{mM} \mathrm{NaCl}, 2 \mathrm{mM}$ EDTA, $0.1 \%$ SDS, protease and phosphatase inhibitor (VWR Life Science Products, Philadelphia, USA) was used to homogenize the frozen brain tissues with a $1 \mathrm{ml}$ syringe, gauge 19 needle. The homogenates were centrifuged for $10 \mathrm{~min}$ at $1,000 \times g$ and the supernatant was collected. The pellet was washed by repeating the homogenizing step again. For Triton-soluble fraction $25 \%$ (w/v) lysis buffer containing $50 \mathrm{mM}$ Tris- $\mathrm{HCl}(\mathrm{pH}$ 7.6), $150 \mathrm{mM} \mathrm{NaCl}, 1 \%$ Triton X-100 (Merck, Darmstadt, Germany) was added to the pellet from the previous centrifugation and pipetted to homogenize the pellet. The homogenates were further centrifuged for $90 \mathrm{~min}$ at $16,000 \times g$ and the supernatant was collected. The pellet was washed by repeating the homogenizing step again. For guanidine $\mathrm{HCl}$ soluble fraction, $25 \%(\mathrm{w} / \mathrm{v})$ lysis buffer containing $5 \mathrm{M}$ guanidine $\mathrm{HCl}$ $(\mathrm{GdnHCl})$ in $50 \mathrm{mM}$ Tris-HCl (EMD-Millipore, Darmstadt, Germany) was added 
to the pellet obtained after centrifugation of the Triton-soluble fraction and pipetted to homogenize the pellet.

Immunostaining. The detailed procedure for immunohistochemistry was described in previous studies ${ }^{62,63}$. In short, coronal sections ( $30 \mu \mathrm{m}$ thickness) were mounted on poly-L lysine coated slides (Thermo Fisher Scientific, Waltham, MA, USA). The coronal section was antigen retrieved by citric acid buffer $(10 \mathrm{mM}$ citric acid, $\mathrm{pH} 6.0,0.05 \%$ Tween-20) at $80^{\circ} \mathrm{C}$ for $30 \mathrm{~min}$, then blocked with $3 \%$ BSA in PBS containing $0.5 \%$ Triton X-100 for $1 \mathrm{~h}$. For immunofluorescence, the coronal section was probed with the primary antibodies: a mixture of $6 \mathrm{E} 10$ (1:2000, SIG39320, BioLegend, San Diego, CA, USA) and 4G8 (1:2000, SIG-39220, BioLegend, San Diego, CA, USA) or polyclonal TDP- 43 oligomer antibody, poly TDP-O 22 (1:1000). The secondary antibody used for $4 \mathrm{G} 8$ and $6 \mathrm{E} 10$ is goat anti-mouse Alexa Fluor 488 (A28175, 1:1000, Invitrogen, Camarillo, CA, USA) and for poly TDP-O is goat anti-rabbit Alexa Fluor 594 (A-11012, 1:1000, Invitrogen, Camarillo, CA USA). Fluorescence images were taken by Aperio FL Digital Pathology Scanner (Leica Biosystem, Mannheim, Germany). The signals were measured by ImageJ (NIH, Bethesda, MD, USA) and a given background threshold was subtracted. The background intensity threshold was fixed and applied to all sections. For immunohistochemistry, rabbit anti-ionized calcium-binding adaptor molecule 1 (Iba1, 1:1000, 091-19741, Wako, Japan) antibody was used. The Ibal signal was then developed using an immunohistochemistry kit (Super Sensitive Polymer-HRP IHC Detection System, Biogenex, Fremont, CA, USA) and the secondary antibody $(1: 1,000)$ provided in the kit. The $3,3^{\prime}$-diaminobenzidine (DAB) was used as the substrate to develop immunohistochemistry signal. To analyze protein expression level, the slide images were taken by Aperio AT2 Digital Pathology Scanner (Leica Biosystem, Mannheim, Germany). All of the quantitative signals were obtained by calculating the signal intensities within the area that were higher than a background threshold with the aid of ImageJ 1.8.0_60 (NIH, USA). To prevent the injection injury effect, the brain slice from the injection side anterior to posterior $0.5 \mathrm{~mm}$ would not be used for quantification. The intensity threshold of background was fixed and applied to all images.

Co-Immunoprecipitation (IP). Co-IP was performed to examine the interaction between A $\beta$ and TDP-43. The buffer and TDP-43 injected APP/PS1 $\Delta$ E9 mice brain samples were taken for analysis as described in brain tissue preparation and fractionation. The extracellular-enriched, Triton-soluble, and $\mathrm{GdnHCl}$ fractions were taken for BCA assay to measure the total protein concentration. Two hundred micrograms of the sample was used for co-IP and $100 \mu \mathrm{g}$ for the input. The fractions were diluted with filtered TBS-T to a total volume of $200 \mu \mathrm{l}$. Two microliters of mouse monoclonal 4G8 antibody against A $\beta$ aa. 17-24 (SIG-39220, BioLegend, San Diego, CA, USA) and $2 \mu \mathrm{l}$ of mouse monoclonal 6 E10 antibody against $A \beta$ aa. 1-17 (SIG-39320, BioLegend, San Diego, CA, USA) was added to the samples for IP study. Mouse IgG2a (mabg2a-ctrlm, InvioGen, San Diego, California, USA) was served as IgG control for this experiment. The samples with the antibodies were mixed overnight in a vertical rotator (F1-10 program, ELMI, Intelli Mixer RM-2L). On the second day, $10 \mu \mathrm{l}$ of Protein A Mag (Sepharose Xtra, $1 \mathrm{ml}$, GE healthcare, Life sciences, Missisauga, Canada) was added to the sample and mixed in the vertical rotator for about $4 \mathrm{~h}$ in $4{ }^{\circ} \mathrm{C}$. Then, the samples were washed with TBS-T for three times in a magnetic rack (20-400. Magna GrIP rack, EMD Millipore, Darmstadt, Germany) and eluted with a $6 x$ loading buffer (25\% glycerol, $50 \mathrm{mM}$ Tris-base, $4 \% \mathrm{SDS}$, and $0.04 \% \beta$ - mercaptoethanol), then heated for $10 \mathrm{~min}$ at $95^{\circ} \mathrm{C}$. Next, the samples were subjected to western blot with gradient gels (NuPAGE 4-12\% Bis-Tris Gel 1.0 mm*15 well, Invitrogen, Carlsbad, CA) in mini gel tanks (A25977, Thermo Fisher Scientific, Waltham, MA, USA) using MES running buffer (B0002, 20x Bolt, MES SDS running buffer, Thermo Fisher Scientific, Waltham, MA, USA) and transferred to PVDF membrane (Amersham Hybond P 0.45, GE healthcare, Chicago, Illinois, USA). The membrane was blocked with $5 \%$ non-fat milk and the TDP-43 antibodies, a mixture of rabbit polyclonal TDP-43 antibody (1:1000, 10782-2-AP, Proteintech, Rosemount, USA) and TDP-43 C-terminal antibody (1:1000, 12892-1-AP, Proteintech, Rosemount, USA), were applied for detection. The images were obtained by Imagequant LAS4000 system (GE Healthcare, Life sciences, Hungary).

Western blot for $\mathbf{A} \boldsymbol{\beta}$ in mice. Western blot was performed to observe the changes in $\mathrm{A} \beta$ assembly after injecting TDP-43 to the APP/PS1 $\triangle \mathrm{E} 9$ mice. The concentration of the extracellular-enriched fraction and Triton-soluble fraction were determined and adjusted to the same protein concentration $(48 \mu \mathrm{g}$ in $20 \mu \mathrm{L})$. The samples were mixed with $6 \mathrm{x}$ loading buffer and heated in $95^{\circ} \mathrm{C}$ for $10 \mathrm{~min}$, then loaded into a $4-15 \%$ Tris-glycine gel and resolved at $100 \mathrm{~V}$ for $120 \mathrm{~min}$. The separated proteins were transferred to a PVDF membrane (Amersham Hybond P 0.45, GE healthcare, Chicago, Illinois, USA) and blocked with 5\% non-fat milk. The mouse monoclonal 6E10 antibody (1:5000, SIG-39320, BioLegend, San Diego, CA, USA) and mouse monoclonal 4G8 antibody (1:5000, SIG-39220, BioLegend, San Diego, CA, USA) were mixed and used for $A \beta$ detection. A mouse monoclonal GAPDH antibody (1:5000, Proteintech, Rosemont, IL, USA) was used for loading control. The secondary antibody used for 6E10/4G8 mixture is goat anti-mouse IgG-HRP (GTX213111-01, Genetex, CA, USA) and for GAPDH is goat anti-rabbit IgG-HRP (GTX213110-01, Genetex, CA, USA). Immobilon Western
Chemiluminescent HRP substrate (Merck, Darmstadt, Germany) was used for developing. The detection was carried out with Imagequant LAS4000 system (GE Healthcare, Life sciences, Hungary) and analyzed using Image (NIH, Bethesda, MD, USA).

Human brain staining. All of the human tissue-related procedures and usage were approved by the Human Subjects Research Ethics, Academia Sinica, Taiwan. Participants were recruited and consented in the Alzheimer's Disease Center at University of California, Davis, Sacramento, California. To investigate the interaction of the TDP-43 oligomer and A $\beta$ in Alzheimer's disease patient, the $5 \mu \mathrm{m}$ thickness paraffin-embedded sections were deparaffinized with xylene. To remove xylene and rehydrate the slides, the specimens were washed through graded ethanol in water and ending with pure $\mathrm{ddH}_{2} \mathrm{O}$. Then, the specimens were immersed in citric acid buffer ( $10 \mathrm{mM}$ citric acid, $\mathrm{pH} 6.0,0.05 \%$ Tween-20) and heated for antigen retrieval at $80{ }^{\circ} \mathrm{C}$ for $30 \mathrm{~min}$, and then blocked with $3 \%$ BSA in PBS with $0.5 \%$ Triton X-100 for $1 \mathrm{~h}$. To observe the amyloid plaque and TDP-43, TDP-43 C-terminal antibody (1:500, 12892-1-AP, Proteintech, Rosemount, USA), poly TDP-O ${ }^{1}$ (1:1000), A $\beta$ antibody (6E10 (1:2000, SIG-39320, BioLegend, San Diego, CA, USA)/ 4 G8 (1:2000, SIG-39220, BioLegend, San Diego, CA, USA)), Aß40-specific antibody 11A50 (1:500, SIG-39140, BioLegend, San Diego, CA, USA), A 342 -specific antibody 12F4 (1:500, SIG-39142, BioLegend, San Diego, CA, USA) and neuronal marker MAP-2 (1:500, 188004, Synaptic systems, Goettingen, Saxony, Germany) were applied to the slides for $2 \mathrm{~h}$ in room temperature. After that, the primary antibodies were washed out by PBS/0.5\% Triton X-100 and probed with the secondary antibodies for $2 \mathrm{~h}$ in room temperature. The secondary antibodies used were goat anti-rabbit Alexa Fluor 594 (A-11012, 1:1000, Invitrogen, Camarillo, CA, USA) for TDP-43 C-terminal antibody and poly TDP-O, goat anti-mouse Alexa Fluor 488 (A28175, 1:1000, Invitrogen, Camarillo, CA, USA) for $\mathrm{A} \beta$, and goat anti-guinea pig Alexa Fluor 647 (A-21450, 1:1000, Invitrogen, Camarillo, CA, USA) for MAP-2. Subsequently, the nuclei were stained with Hoechst Stain solution $(1: 1000,0.5 \mathrm{ng} / \mathrm{ml}, \mathrm{H} 6024$, Sigma-Aldrich, St. Louis, Missouri, USA). Lastly, glycerol gelatin (GG1-10X15ML, Sigma-Aldrich, St. Louis, MO, USA) was applied to mount each tissue slides. After the staining procedure, the human brain slide images were captured by Aperio CS2 Digital Pathology Scanner (Leica Biosystem).

Reporting summary. Further information on research design is available in the Nature Research Reporting Summary linked to this article.

\section{Data availability}

Data for figures of this manuscript and supplementary information are available in the source data file. Other data from the corresponding authors upon request. Source data are provided with this paper.

Received: 16 September 2019; Accepted: 30 October 2020; Published online: 23 November 2020

\section{References}

1. Bloom, G. S. Amyloid-beta and tau: the trigger and bullet in Alzheimer disease pathogenesis. JAMA Neurol. 71, 505-508 (2014).

2. Querfurth, H. W. \& LaFerla, F. M. Alzheimer's disease. N. Engl. J. Med. 362, 329-344 (2010).

3. Dahlgren, K. N. et al. Oligomeric and fibrillar species of amyloid-beta peptides differentially affect neuronal viability. J. Biol. Chem. 277, 32046-32053 (2002).

4. McGowan, E. et al. Abeta42 is essential for parenchymal and vascular amyloid deposition in mice. Neuron 47, 191-199 (2005).

5. Selkoe, D. J. \& Podlisny, M. B. Deciphering the genetic basis of Alzheimer's disease. Annu. Rev. Genomics Hum. Genet. 3, 67-99 (2002).

6. Kuperstein, I. et al. Neurotoxicity of Alzheimer's disease Abeta peptides is induced by small changes in the Abeta42 to Abeta40 ratio. EMBO J. 29, 3408-3420 (2010).

7. Haass, C. \& Selkoe, D. J. Soluble protein oligomers in neurodegeneration: lessons from the Alzheimer's amyloid beta-peptide. Nat. Rev. Mol. Cell Biol. 8 101-112 (2007).

8. Eichner, T. \& Radford, S. E. A diversity of assembly mechanisms of a generic amyloid fold. Mol. Cell 43, 8-18 (2011).

9. LaFerla, F. M., Green, K. N. \& Oddo, S. Intracellular amyloid-beta in Alzheimer's disease. Nat. Rev. Neurosci. 8, 499-509 (2007).

10. Neumann, M. et al. Ubiquitinated TDP-43 in frontotemporal lobar degeneration and amyotrophic lateral sclerosis. Science 314, 130-133 (2006).

11. King, A. et al. Abnormal TDP-43 expression is identified in the neocortex in cases of dementia pugilistica, but is mainly confined to the limbic system when identified in high and moderate stages of Alzheimer's disease. Neuropathology 30, 408-419 (2010). 
12. Kadokura, A., Yamazaki, T., Lemere, C. A., Takatama, M. \& Okamoto, K. Regional distribution of TDP-43 inclusions in Alzheimer disease (AD) brains: their relation to AD common pathology. Neuropathology 29, 566-573 (2009).

13. Josephs, K. A. et al. Staging TDP-43 pathology in Alzheimer's disease. Acta Neuropathol. 127, 441-450 (2014).

14. Tremblay, C., St-Amour, I., Schneider, J., Bennett, D. A. \& Calon, F. Accumulation of transactive response DNA binding protein 43 in mild cognitive impairment and Alzheimer disease. J. Neuropathol. Exp. Neurol. 70, 788-798 (2011).

15. Nelson, P. T. et al. Limbic-predominant age-related TDP-43 encephalopathy (LATE): consensus working group report. Brain 142, 1503-1527 (2019).

16. Sun, Y. \& Chakrabartty, A. Phase to phase with TDP-43. Biochemistry 56, 809-823 (2017).

17. Wang, H. Y., Wang, I. F., Bose, J. \& Shen, C. K. Structural diversity and functional implications of the eukaryotic TDP gene family. Genomics 83, 130-139 (2004).

18. Buratti, E. et al. Nuclear factor TDP-43 and SR proteins promote in vitro and in vivo CFTR exon 9 skipping. EMBO J. 20, 1774-1784 (2001).

19. Chang, C. K. et al. The N-terminus of TDP-43 promotes its oligomerization and enhances DNA binding affinity. Biochem. Biophys. Res. Commun. 425 219-224 (2012).

20. Afroz, T. et al. Functional and dynamic polymerization of the ALS-linked protein TDP-43 antagonizes its pathologic aggregation. Nat. Commun. 8, 45 (2017).

21. Igaz, L. M. et al. Expression Of TDP-43 C-terminal fragments in vitro recapitulates pathological features of TDP-43 proteinopathies. J. Biol. Chem. 284, 8516-8524 (2009).

22. Fang, Y. S. et al. Full-length TDP-43 forms toxic amyloid oligomers that are present in frontotemporal lobar dementia-TDP patients. Nat. Commun. 5, 4824 (2014).

23. Caccamo, A., Magri, A. \& Oddo, S. Age-dependent changes in TDP-43 levels in a mouse model of Alzheimer disease are linked to A beta oligomers accumulation. Mol. Neurodegener. 5, 51 (2010).

24. Herman, A. M., Khandelwal, P. J., Rebeck, G. W. \& Moussa, C. E. Wild type TDP-43 induces neuro-inflammation and alters APP metabolism in lentiviral gene transfer models. Exp. Neurol. 235, 297-305 (2012).

25. Lippa, C. F. et al. Transactive response DNA-binding protein 43 burden in familial Alzheimer disease and Down syndrome. Arch. Neurol. 66, 1483-1488 (2009).

26. Biancalana, M. \& Koide, S. Molecular mechanism of Thioflavin-T binding to amyloid fibrils. Biochim. Biophys. Acta 1804, 1405-1412 (2010).

27. Kayed, R. et al. Common structure of soluble amyloid oligomers implies common mechanism of pathogenesis. Science 300, 486-489 (2003).

28. Kayed, R. et al. Fibril specific, conformation dependent antibodies recognize a generic epitope common to amyloid fibrils and fibrillar oligomers that is absent in prefibrillar oligomers. Mol. Neurodegener. 2, 18 (2007).

29. Wu, J. W. et al. Fibrillar oligomers nucleate the oligomerization of monomeric amyloid beta but do not seed fibril formation. J. Biol. Chem. 285, 6071-6079 (2010).

30. Wang, Y. T. et al. The truncated C-terminal RNA recognition motif of TDP43 protein plays a key role in forming proteinaceous aggregates. J. Biol. Chem. 288, 9049-9057 (2013).

31. Bliss, T. V. \& Collingridge, G. L. A synaptic model of memory: long-term potentiation in the hippocampus. Nature 361, 31-39 (1993).

32. Puzzo, D. et al. Amyloid-beta peptide inhibits activation of the nitric oxide/ cGMP/cAMP-responsive element-binding protein pathway during hippocampal synaptic plasticity. J. Neurosci. 25, 6887-6897 (2005).

33. Lesne, S. et al. A specific amyloid-beta protein assembly in the brain impairs memory. Nature 440, 352-357 (2006).

34. Perlenfein, T. J., Mehlhoff, J. D. \& Murphy, R. M. Insights into the mechanism of cystatin $\mathrm{C}$ oligomer and amyloid formation and its interaction with betaamyloid. J. Biol. Chem. 292, 11485-11498 (2017).

35. Li, X. et al. Mechanisms of transthyretin inhibition of beta-amyloid aggregation in vitro. J. Neurosci. 33, 19423-19433 (2013).

36. Bitan, G. et al. Amyloid beta -protein (Abeta) assembly: Abeta 40 and Abeta 42 oligomerize through distinct pathways. Proc. Natl Acad. Sci. USA 100 , 330-335 (2003).

37. Bernstein, S. L. et al. Amyloid- $\beta$ protein oligomerization and the importance of tetramers and dodecamers in the aetiology of Alzheimer's disease. Nat. Chem. 1, 326-331 (2009)

38. Chen, Y. R. \& Glabe, C. G. Distinct early folding and aggregation properties of Alzheimer amyloid-beta peptides A beta 40 and A beta 42 - Stable trimer or tetramer formation by A beta 42. J. Biol. Chem. 281, 24414-24422 (2006).

39. Fezoui, Y. \& Teplow, D. B. Kinetic studies of amyloid beta-protein fibril assembly. Differential effects of alpha-helix stabilization. J. Biol. Chem. 277, 36948-36954 (2002).
40. Zatz, M. \& Starling, A. Calpains and disease. N. Engl. J. Med 352, 2413-2423 (2005).

41. Yamashita, T. et al. A role for calpain-dependent cleavage of TDP-43 in amyotrophic lateral sclerosis pathology. Nat. Commun. 3, 1307 (2012).

42. LaClair, K. D. et al. Depletion of TDP-43 decreases fibril and plaque betaamyloid and exacerbates neurodegeneration in an Alzheimer's mouse model. Acta Neuropathol. 132, 859-873 (2016).

43. Meraz-Rios, M. A., Toral-Rios, D., Franco-Bocanegra, D., Villeda-Hernandez, J. \& Campos-Pena, V. Inflammatory process in Alzheimer's Disease. Front. Integr. Neurosci. 7, 59 (2013).

44. Flores, J. et al. Caspase-1 inhibition alleviates cognitive impairment and neuropathology in an Alzheimer's disease mouse model. Nat. Commun. 9, 3916 (2018).

45. O’Brien, R. J. \& Wong, P. C. Amyloid precursor protein processing and Alzheimer's disease. Annu. Rev. Neurosci. 34, 185-204 (2011).

46. Cabrejo, L. et al. Phenotype associated with APP duplication in five families. Brain 129, 2966-2976 (2006).

47. Gouras, G. K. et al. Intraneuronal Abeta42 accumulation in human brain. Am. J. Pathol. 156, 15-20 (2000).

48. Gyure, K. A., Durham, R., Stewart, W. F., Smialek, J. E. \& Troncoso, J. C. Intraneuronal abeta-amyloid precedes development of amyloid plaques in Down syndrome. Arch. Pathol. Lab. Med. 125, 489-492 (2001).

49. Pasternak, S. H. et al. Presenilin-1, nicastrin, amyloid precursor protein, and $\gamma$-secretase activity are co-localized in the lysosomal membrane. J. Biol. Chem. 278, 26687-26694 (2003).

50. Leibiger, C. et al. TDP-43 controls lysosomal pathways thereby determining its own clearance and cytotoxicity. Hum. Mol. Genet. 27, 1593-1607 (2018).

51. Wang, I. F. et al. Autophagy activators rescue and alleviate pathogenesis of a mouse model with proteinopathies of the TAR DNA-binding protein 43. Proc. Natl Acad. Sci. USA 109, 15024-15029 (2012).

52. Barmada, S. J. et al. Autophagy induction enhances TDP43 turnover and survival in neuronal ALS models. Nat. Chem. Biol. 10, 677-685 (2014).

53. $\mathrm{Hu}, \mathrm{X}$. et al. Amyloid seeds formed by cellular uptake, concentration, and aggregation of the amyloid-beta peptide. Proc. Natl Acad. Sci. USA 106, 20324-20329 (2009).

54. Buckig, A., Tikkanen, R., Herzog, V. \& Schmitz, A. Cytosolic and nuclear aggregation of the amyloid beta-peptide following its expression in the endoplasmic reticulum. Histochem. Cell Biol. 118, 353-360 (2002).

55. Fonte, V. et al. Interaction of intracellular beta amyloid peptide with chaperone proteins. Proc. Natl Acad. Sci. USA 99, 9439-9444 (2002).

56. Yoon, E. J. et al. Intracellular amyloid beta interacts with SOD1 and impairs the enzymatic activity of SOD1: implications for the pathogenesis of amyotrophic lateral sclerosis. Exp. Mol. Med. 41, 611-617 (2009).

57. Lustbader, J. W. et al. ABAD directly links Abeta to mitochondrial toxicity in Alzheimer's disease. Science 304, 448-452 (2004).

58. Shankar, G. M. et al. Amyloid-beta protein dimers isolated directly from Alzheimer's brains impair synaptic plasticity and memory. Nat. Med. 14, 837-842 (2008).

59. Kuo, P. H., Doudeva, L. G., Wang, Y. T., Shen, C. K. J. \& Yuan, H. S. Structural insights into TDP-43 in nucleic-acid binding and domain interactions. Nucleic Acids Res. 37, 1799-1808 (2009).

60. Liao, Y. H. \& Chen, Y. R. A novel method for expression and purification of authentic amyloid-beta with and without (15)N labels. Protein Expr. Purif. 113, 63-71 (2015)

61. Lee, M. C. et al. Zinc ion rapidly induces toxic, off-pathway amyloid-beta oligomers distinct from amyloid-beta derived diffusible ligands in Alzheimer's disease. Sci. Rep. 8, 4772 (2018).

62. Shih, Y. H. et al. Hypertension impairs hippocampus-related adult neurogenesis, CA1 neuron dendritic arborization and long-term memory. Neuroscience 322, 346-357 (2016).

63. Lee, M. H. et al. Zfra restores memory deficits in Alzheimer's disease tripletransgenic mice by blocking aggregation of TRAPPC6ADelta, SH3GLB2, tau, and amyloid beta, and inflammatory NF-kappaB activation. Alzheimers Dement. (N. Y) 3, 189-204 (2017).

\section{Acknowledgements}

We thank research founding from Career Development Grant, Academia Sinica (ASCDA-106-L01), Genomics Research Center, Academia Sinica, and Ministry of Science and Technology, Taiwan (MOST 108-2113-M-001-027). The work was supported in part by the U.S. National Institute on Aging grant P30 AG10129 (University of California Davis Alzheimer's Disease Research Center). We thank Dr. Hanna S. Yuan, Institute of Molecular Biology, Academia Sinica for providing pQE30 plasmids and E. coli strain M15. We thank Dr. Shu-Chuan Jao of the Biophysics Core Facility, funded by Academia Sinica Core Facility and Innovative Instrument Project (AS-CFII108-111), and Mr. Hong-Chang Chu in Dr. Ying-Da Wu's laboratory at Genomics Research Center, Academia Sinica for providing technical assistance of biolayer interferometry experiments. 
We thank TEM Core Facility, Academia Sinica, for assisting TEM imaging. We thank National Laboratory Animal Center (NLAC), NARLabs, Taiwan, for technical support in contract breeding and testing services and Dr. Sin-Jhong Cheng of the NeuroElectrophysiology Core, Neuroscience Program of Academia Sinica (NPAS) for providing technical support in LTP studies.

\section{Author contributions}

L.H.T. designed the biochemical studies; Y.H.S. designed the animal studies; L.H.T., T.Y.C., and Y.T.L. performed the biochemical studies; Y.H.S. and K.G. performed the animal studies; Y.H.S., K.G., and P.S.C. performed the immunostaining of human brain tissues; W.W.C. performed western blot of human brain tissues. Y.S.F cloned the plasmid TDP-43 1-265; L.W.J. provided clinical assessment and human brain tissues; Y.H.S., L.H.T., K.G., W.W.C., T.Y.C., and Y.R.C. analyzed the data and wrote the manuscript; and Y.R.C. conducted the research direction.

\section{Competing interests}

The authors declare no competing interests.

\section{Additional information}

Supplementary information is available for this paper at https://doi.org/10.1038/s41467020-19786-7.

Correspondence and requests for materials should be addressed to Y.-R.C.
Peer review information Nature Communications thanks Shin Kwak and the other, anonymous, reviewer(s) for their contribution to the peer review of this work. Peer reviewer reports are available.

Reprints and permission information is available at http://www.nature.com/reprints

Publisher's note Springer Nature remains neutral with regard to jurisdictional claims in published maps and institutional affiliations.

cc (i) Open Access This article is licensed under a Creative Commons Attribution 4.0 International License, which permits use, sharing, adaptation, distribution and reproduction in any medium or format, as long as you give appropriate credit to the original author(s) and the source, provide a link to the Creative Commons license, and indicate if changes were made. The images or other third party material in this article are included in the article's Creative Commons license, unless indicated otherwise in a credit line to the material. If material is not included in the article's Creative Commons license and your intended use is not permitted by statutory regulation or exceeds the permitted use, you will need to obtain permission directly from the copyright holder. To view a copy of this license, visit http://creativecommons.org/ licenses/by/4.0/.

(C) The Author(s) 2020 